\title{
GEOCHEMISTRY AND PETROGENESIS OF THE CALC-ALKALINE GRANITOIDS FROM THE NORTHERN ARABIAN-NUBIAN SHIELD. A CASE STUDY FROM WADI EL-AKHDER GRANITOIDS, SOUTHERN SINAI, EGYPT
}

\author{
Moustafa Mohamed Mogahed \\ Geology Department, Benha University-Egypt, P.O. Box 13518, Benha, Egypt; \\ E-mail: mustafa.ahmed01@fsc.bu.edu.eg
}

\begin{abstract}
I-type granitoids emplaced during pre-collision stages of the Neoproterozoic Pan-African Orogeny, are widely distributed in the southern Sinai Peninsula and constituting 30\% of the basement outcrops. Petrological, geochemical, and mineral chemistry data are presented for the I-type, Wadi El-Akhder granitic suite (AGR), in order to determine their origin and geotectonic implications. The tonalitegranodiorites of Wadi El-Akhder have geochemical characteristics of medium-K calc-alkaline, metaluminous to mildly peraluminous granitoids formed in an island-arc environment, which are conformable with well-known Egyptian older granitoids rocks $\left(\mathrm{G}_{1}\right)$. They have low $\mathrm{Al}_{2} \mathrm{O}_{3}, \mathrm{FeO}^{*}, \mathrm{MgO}$, $\mathrm{MnO}, \mathrm{CaO}, \mathrm{TiO}_{2}, \mathrm{Sr}, \mathrm{Ba}$, and $\mathrm{V}$ contents, but possess high contents of $\mathrm{Na}_{2} \mathrm{O}, \mathrm{K}_{2} \mathrm{O}, \mathrm{Nb}, \mathrm{Zr}, \mathrm{Th}$, and $\mathrm{Rb}$. The studied AGR are generally characterized by enrichment of LILE and LREE and depletion of HFSE relative to N-MORB values, and follow assimilation and fractional crystallization (AFC) trends indicative of extensive crustal contamination of magma derived from a mantle source. The studied granitic rocks show high $\mathrm{CaO} / \mathrm{Na}_{2} \mathrm{O}$ ratios $(0.82-1.54)$ being decrease from tonalite to granodiorites, whereas they have also high $\mathrm{Al}_{2} \mathrm{O}_{3} / \mathrm{TiO}_{2}$ ratios (16.69-27.61) increasing toward the granodiorites. These features indicate that emplacement of the granitoid were strongly affected by magma mixing of felsic and mafic melts. The chemical characteristics of the AGR are remarkably similar to those of subduction-related granitoids from the Arabian-Nubian Shield (ANS).
\end{abstract}

Keywords: Southern Sinai; Arabian-Nubian Shield; I-type granite; magma mixing.

\section{INTRODUCTION}

Basement outcrops in Sinai Peninsula, comprise the northernmost part of the ANS (Fig. 1). ANS was evolved through four main stages. The oceanic, island arcs and plutonic rocks were formed during the subduction stage ( 950-850 Ma, Bentor 1985; Genna et al. 2002; Johnson and Woldehaimanot 2003; Kroner et al. 1990; Stern and Manton 1987). The continental collision ( $\sim 850-650 \mathrm{Ma}$, Avigad and Gvirtzman 2009; Be'eri-Shlevin et al. 2009) resulting from continuing convergence between East and West Gondwana to form the East African Orogen (Stern 1994). The post-collision stage ( 650-580 Ma) marks stabilization of the shield and is characterized by widespread calc-alkaline magmatism, mainly of intermediate to felsic composition and the occurrence of a vast peneplain (Avigad and Gvirtzman 2009; Avigad et al. 2005). The last stage ( Gass 1981; Roobol et al. 1983) representing late to post-orogenic, intracratonic and within-plate magmatism is characterized by the severe igneous activity where alkaline to peralkaline granites were emplaced.

The Precambrian rocks occuring at the southern part of the Sinai Peninsula can be divided into metamorphic complexes (old continental gneisses), intermediate to acidic subduction-related volcanics and metasediments (island arcs), subduction-related granites (older granites), post-orogenic granites (younger granites), rift-related volcanics and finally alkaline anorogenic granites (El-Tokhi et al. 2009).

Understanding the origin of the granitoid rocks has important implications for the continental crust growth in general and for the ANS in particular. The Egyptian granitoids are broadly subdivided into three main cycles: the first cycle of synorogenic granitoids (Gray and Shaitian types) with ages ranging between $626 \pm 2$ and $780 \pm 7$ Ma (Dixon 1981; Stern and Manton 1987; Struchio et al. 1982). The second cycle of 


\section{Mogahed, M. M.}

younger granitoids (late orogenic granites) which were dated in Sinai and Egypt at 560-600 Ma (Bielski et al. 1979). Moreover, the epizonal granites and mesozonal granodiorites were recorded as the major varieties of plutonic rocks in the North Eastern Desert of Egypt (Stern et al. 1984). The mesozonal granodiorites represent the older variety and exhibiting calc-alkaline affinity. The epizonal granites are younger, representing anorogenic magmatic pulse unrelated to subduction-zone magmatism, related to the major episode of NW-SE to NS directed rifting in northernmost Afro-Arabia (Stern 1985) and generally known as pink, or Pan-African granites (Stern et al. 1984).

Generally, the granitoid rocks in Sinai Peninsula exhibiting evolution trend from calc-alkaline through alkaline to per-alkaline granitoids. Calc-alkaline granitoids are older and comprise diorite-tonalitegranodiorite suites (Granite I) and Alkaline to per-alkaline granitoids are younger and comprise calcalkaline younger biotite granite, younger post-tectonic granite or late-tectonic monzogranite (e.g., AbdelRahman and El-Kibbi 2001; Ahmed et al. 1993; El-Mettwaly et al. 1992; El-Sheshtawi et al. 1993; Furnes et al. 1996; Moghazi 1999; Moghazi 2002; Mohamed et al. 1994). This trend is similar to that recognized among granitic rocks in the Eastern Desert of Egypt.

Although the granitoid rocks from the ANS have been subjected to numerous investigations concerning their petrogenesis and geotectonic evolution, but much controversy still persists on their source regions and the role of fractional crystallization against crustal anatexis (e.g., Beyth et al. 1994; Stern and Gottfried 1986). Wadi El-Akhder area, southwestern Sinai, Egypt (Fig. 1), represents a good example for resolving these issues, as it constitutes the biggest batholith of the older granitic rock in southeastern Sinai (Bentor 1985).

Fig. 1: (a) Map of southern Sinai, Egypt (Gahlan et al., 2017) exhibiting the location of the studied area.

b) Geological map of Wadi El-Akhder granitic rocks.

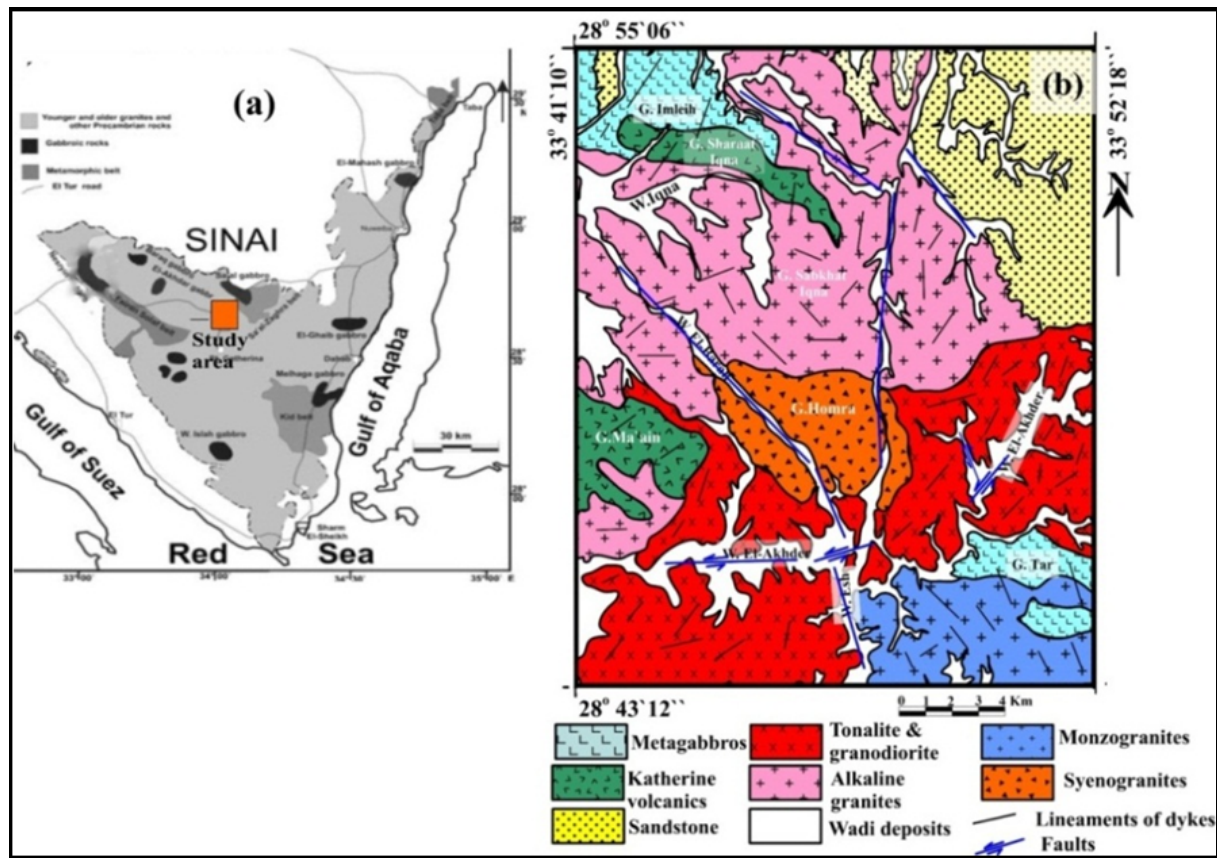

Heikal et al. (1985) mentioned the granitoid rocks around Wadi El-Sheikh that comprising the study area as two groups older and younger granitoids. Stern and Hedge (1985) gave the age of $632 \mathrm{Ma}$ (U-Pb method) and $780 \mathrm{Ma}(\mathrm{Rb}-\mathrm{Sr}$ method) for the older granitoids of Heikal et al. (1985) that intruded into the gneisses of Feiran-Solaf metamorphic belt. The calc-alkaline granitoids in the study area are related to compressional environment, Moreover, El-Mettwaly and Mashaal (1989) and Mashaal et al. (1999) showed that the granitoids rocks of Wadi Ager, which occur beyond the western border of the mapped area, are calc-alkaline, I-type orogenic granodiorite that emplaced under compressional environment.

The present study introduces new data on the petrology, geochemistry and mineral chemistry of the older granitic rocks in Wadi El-Akhder area, southwestern Sinai, Egypt in order to elucidate their tectonic environments and to shed some light on their origin. 
Geochemistry and petrogenesis of the calc-alkaline Granitoids

\section{GEOLOGIC SETTINGS}

Wadi El-Akhder area is located at the southwestern part of the Sinai Peninsula and is bounded by the Feiran-Solaf metamorphic belt from the west and Katherina Ring Complex from the east (Fig. 1). The area is bounded by latitudes $28^{\circ} 43^{\prime} 12^{\prime \prime}$ and $28^{\circ} 55^{\prime} 06^{\prime \prime} \mathrm{N}$, and longitudes $33^{\circ} 41^{\prime} 10^{\prime \prime}$ to $33^{\circ} 52^{\prime} 18^{\prime \prime} \mathrm{E}$, covering about $550 \mathrm{~km}^{2}$ (Fig. 1). It is dissected by a high density of faults due to its proximity to both the NW-trending faults of the Gulf of Suez system and the NNE-trending faults of the Gulf of Aqaba system. The main map-scale units in the area include metagabbros, subduction-related syn-tectonic granitoids (AGR), monzogranites, Katherine volcanics, alkaline granites, syenogranites, and Phanerozoic sedimentary cover (Fig. 1). The metagabbroic rocks crop out in the northwestern and southeastern corners of the studied area (Fig. 1), are intruded by tonalites, monzogranites (Fig. 2a) and Katherine volcanics. On the other hand, the Katherine volcanics crop out at G. Sharaat Iqna and G. Maa in (Fig. 1), are generally classified into trachytes, rhyodacites and rhyolites and intruded by alkaline granites (Fig. 2b).

The chronological sequence of the different rock units in the mapped area starts with metagabbros followed by the emplacement of AGR during the late phase of the Pan-African Orogeny. This was followed by the intrusion of monzogranite. Afterwards, extrusive Katherina volcanics were erupted and syenogranite and alkali granites were intruded.

The investigated syn-tectonic granitoids (equivalent to G1 Egyptian granites of Hussein et al., 1982) are represented in Wadi El-Akhder area by tonalites and granodiorites. The exposures of these granitoids are limited compared to the other granitic types. They cover the area around the northern and southern flanks of Wadi El-Akhder (Fig. 1) occupying an area of about $130 \mathrm{~km}^{2}$ (about $40 \%$ ) of the whole surface of the study area. The tonalites crop out along the central parts of Wadi El-Akhder and representing the core of AGR, whereas the granodiorites crop out at the entrance of Wadi El-Akhder and extended to the east beyond the limits of the mapped area intruding the well-known Feiran-Solaf gneisses.

Wadi El-Akhder granitoids are coarse- to medium-grained, with low to moderate relief and normally dark gray color. They are highly jointed and show bouldery weathering. Sometimes they exhibit weak gneissosity, which is commonly observed along the contact with gneissic rocks. Generally, these rocks have xenoliths of the pre-existing gneisses and amphibolites which mainly striking N50-60 ${ }^{\circ} \mathrm{E}$ (Fig. 2c). The granitic rocks are intruded by monzogranites alkali granites with sharp contact (Fig. 2d). In some places, AGR occur as xenoliths (Fig. 2e) or roof pendant within the monzogranites and alkali granites (Fig. 2f). Moreover, the area is dissected by numerous felsic and mafic dykes. These dykes striking N-S, NNE-SSW, NE-SW and E-W. The felsic dykes are represented by microgranite porphyry, granophyre, porphyritic rhyolite and aplite with N-S and NNE-SSW predominant trends. The mafic dykes are mostly basaltic in composition acquiring commonly NE-SW and E-W directions.

\section{PETROGRAPHY OF THE STUDIED GRANITIC ROCKS}

These granitoids exhibit a wide range of mineralogical composition and textural variations. They are mainly of granodioritic in composition but with locally tonalitic and are characterized by containing mafic minerals ranging from $15 \%$ to $25 \%$ of the whole rock volume and exhibiting different varieties of textures ranging from hypidiomorphic, local gneissose, granoblastic, perthitic to porphyritic.

Microscopically, the granodioritic rocks could be subdivided into biotite granodiorite, gneissose granodiorite and rare adamellite. The biotite granodiorite is composed mainly of plagioclase $(40-50 \%$ by volume), potash feldspars $(10 \%)$, quartz $(30 \%)$ and biotite with rare hornblende (Fig. 3a). Plagioclases are subhedral to euhedral crystals, ranging in composition from andesine to oligoclase with more sodic rims and occur as megacrysts embedded in crushed quartz and feldspar groundmass or occur as inclusions in the perthite. Potash feldspars are mainly represented by perthitic orthoclase and microcline. Quartz occurs both as small discrete grains and intergrown with interstitial microcline. The mafic minerals range from $10-15 \%$ of the whole rock and constitute brown biotite which may be partially chloritized and rarely subhedral to euhedral green hornblende and augite (Fig. 3b). The accessory minerals are iron-oxides, sphene, apatite and zircon (Fig. 3b). The gneissose granodiorite has the same composition of the biotite granodiorite but possess slightly gneissose texture. The adamellite is essentially composed of potash 
Mogahed, M. M.

feldspars (up to $40 \%$ by volume) exhibiting subsolvous texture, plagioclases $(30 \%)$ which are frequently zoned, less quartz (20\%) and equal amounts of biotite and hornblende (Fig. 3c).

The tonalitic varieties of the studied syn-tectonic granitoids are mainly composed of plagioclases, potash feldspars, biotite, hornblende and quartz (Fig. 3d). Plagioclases are more calcic $\left(\mathrm{An}_{64-70}\right)$ whereas potash feldspars occur as subhedral to anhedral orthoclase-perthite and containing biotite inclusions. Brown biotites are generally chloritized and sometimes replacing hornblende crystals. Quartz generally occurs as anhedral grains that is usually form quartz ocelli where the quartz is rimmed by hornblende and/or biotite (Fig. 3d).


Fig. 2: Representative field photographs from the studying area showing, (a) Roof pendant of metagabbros carried by monzogranite. (b) Low-angle contact between the acidic volcanic rocks of Gabal sharat Iqna and alkali granites. (c) Granodiorite from southwestern part of the mapped area exhibiting weak foliation and enclosing old relics of tonalitic gneiss. (d) Low-angle contact between AGR (tonalite) and monzogranite along Wadi Esh. (e) Xenolith of granodiorite in monzogranite, along Wadi El-Akhder. (f) Syenogranite carry roof pendant of pre-existing granodiorite (upper side).



Fig. 3: (a) Biotite-granodiorite showing biotite flakes, hornblende, kaolinized plagioclase and quartz.

(b) Cumulate of brown-biotite, iron oxide, apatite, sphene with altered plagioclase and quartz. (c) Adamellite showing zoned plagioclase, microperthite, biotite, hornblende and quartz. (d) Tonalite exhibiting plagioclase and quartz which is rimmed by hornblende and biotite form quartz ocelli. All images are in crossed-polarized light. Abbreviations: Qz- quartz; Plplagioclase; Per- perthite; $\mathrm{Bi}$ biotite; Hb- hornblende and Ssphene.

\section{ANALYTICAL TECHNIQUES}

Mineral analyses were performed by electron microprobe analysis (EMPA) at the Far Eastern Geologic Institute, Vladivostok of the Russian Academy of Sciences, using a JEOL JXA8100 instrument with three wave spectrometers, completed with an INCA x-sight energy dispersive spectrometer. Corrections for dead-time, atomic number, absorption and fluorescence effects were made, using the computer programs 
Geochemistry and petrogenesis of the calc-alkaline Granitoids

based on the methods of (Sweatman and Long 1969). Representative EMPA data of minerals (amphiboles, biotites and feldspars) are given in Tables (1,2,3 and 4).

After petrographic screening ten samples representing the studied granitic rocks (4 tonalites and 6 granodiorites) exhibiting least alteration has been selected for chemical analysis. Loss on ignition (LOI) and whole-rock chemical analyses were carried out for major oxides, trace elements and rare earth elements (REEs) at the Far Eastern Geologic Institute, Vladivostok of the Russian Academy of Sciences.

Trace-elements and the REEs analyses were carried out by LA-ICP-MS (laser ablation-inductively coupled plasma-mass spectrometry) using an Agilent $7500 \mathrm{~s}$ quadrupole ICP-MS system attached to a New Wave/Merchantek UP213 laser ablation system. The analytical conditions of such element were a beam diameter of $40 \mu \mathrm{m}$ and a $5 \mathrm{~Hz}$ repetition rate. The typical precision and accuracy of these NIST- 610 analyses are $2-5 \%$ for REEs, Ta, Hf, Th, U, Y, Sr and $\mathrm{Nb}$ at the ppm concentration level and from 7 to $10 \%$ for elements such as P, Mn, Fe and Ga (Norman et al. 1998; Norman et al. 1996). Whole-rock major and trace element data are reported in Table 5.

\section{MINERAL CHEMISTRY}

The microprobe analyses for major elements together with the recalculated cations of the amphiboles, biotites and feldspars in the studied granitic rocks are listed in Tables (1-4).

Table 1: Electron microprobe analyses of amphiboles from the studied granitic rocks of Wadi El-Akhder area southwestern Sinai, Egypt.

\begin{tabular}{|c|c|c|c|c|c|c|c|c|}
\hline \multirow{2}{*}{$\begin{array}{l}\text { Rock type } \\
\text { Petro. Name }\end{array}$} & \multicolumn{8}{|c|}{$\begin{array}{c}\text { Syn-tectonic granitoids } \\
\text { Wadi El-Akhder granitic rocks (AGR) }\end{array}$} \\
\hline & \multicolumn{3}{|c|}{ Tonalites } & \multicolumn{5}{|c|}{ Granodiorites } \\
\hline Sample No. & \multicolumn{3}{|c|}{$\mathrm{T} 17 / 1$} & \multicolumn{5}{|c|}{ G19/1 } \\
\hline Amph. Name & \multicolumn{8}{|c|}{ Actinolite } \\
\hline Amph. class. & \multicolumn{8}{|c|}{ Calcic } \\
\hline $\mathrm{SiO}_{2}$ & 47.97 & 47.14 & 47.28 & 48.78 & 48.83 & 50.13 & 51.91 & 51.11 \\
\hline $\mathrm{TiO}_{2}$ & 1.33 & 0.91 & 1.12 & 0.96 & 0.86 & 0.87 & 0.77 & 0.93 \\
\hline $\mathrm{Al}_{2} \mathrm{O}_{3}$ & 8.33 & 9.91 & 10.37 & 8.89 & 9.03 & 8.87 & 5.27 & 6.84 \\
\hline $\mathrm{FeO}^{*}$ & 9.24 & 10.48 & 9.23 & 10.16 & 9.87 & 9.65 & 10.47 & 9.72 \\
\hline $\mathrm{MnO}$ & 0.49 & 0.69 & 0.71 & 0.37 & 0.87 & 0.34 & 0.30 & 0.39 \\
\hline $\mathrm{MgO}$ & 17.11 & 17.41 & 17.41 & 15.97 & 16.41 & 16.71 & 17.61 & 17.51 \\
\hline $\mathrm{CaO}$ & 11.38 & 10.34 & 11.21 & 9.13 & 8.65 & 8.89 & 9.55 & 9.12 \\
\hline $\mathrm{Na}_{2} \mathrm{O}$ & 0.81 & 0.61 & 0.71 & 1.88 & 2.03 & 1.79 & 1.34 & 1.57 \\
\hline $\mathrm{K}_{2} \mathrm{O}$ & 0.41 & 0.33 & 0.41 & 0.62 & 0.51 & 0.23 & 0.19 & 0.41 \\
\hline Total & 97.07 & 97.82 & 98.45 & 96.76 & 97.06 & 97.28 & 97.01 & 97.06 \\
\hline \multicolumn{9}{|c|}{ Formula based on 23 oxygen atoms and assuming a total of 13 cations exclusive of $\mathrm{Ca}, \mathrm{Na}$ and $\mathrm{K}$} \\
\hline $\mathrm{Si}$ & 6.83 & 6.64 & 6.61 & 6.86 & 6.85 & 6.96 & 7.24 & 7.12 \\
\hline $\mathrm{Al}^{-(\mathrm{iv})}$ & 1.17 & 1.36 & 1.39 & 1.14 & 1.15 & 1.04 & 0.76 & 0.88 \\
\hline Sum T site & 8 & 8 & 8 & 8 & 8 & 8 & 8 & 8 \\
\hline $\mathrm{Ti}$ & 0.14 & 0.1 & 0.12 & 0.1 & 0.1 & 0.11 & 0.1 & 0.12 \\
\hline $\mathrm{Al}^{\text {(vi) }}$ & 0.22 & 0.29 & 0.32 & 0.33 & 0.34 & 0.38 & 0.1 & 0.24 \\
\hline $\mathrm{Fe}^{3+}$ & 0.59 & 0.82 & 0.76 & 1.19 & 1.14 & 1.11 & 1.19 & 1.11 \\
\hline $\mathrm{Fe}^{2+}$ & 0.42 & 0.14 & 0.17 & 0.03 & - & - & - & - \\
\hline $\mathrm{Mg}$ & 3.63 & 3.66 & 3.63 & 3.35 & 3.43 & 3.41 & 3.61 & 3.53 \\
\hline Sum C site & 5.00 & 5.01 & 5.00 & 5.00 & 5.01 & 5.01 & 5.00 & 5.00 \\
\hline $\mathrm{Mn}^{2+}$ & 0.06 & 0.08 & 0.08 & 0.04 & 0.10 & 0.04 & 0.04 & 0.05 \\
\hline $\mathrm{Fe}^{2+}$ & 0.10 & 0.27 & 0.14 & 0.09 & 0.10 & 0.17 & 0.17 & 0.17 \\
\hline $\mathrm{Ca}$ & 1.74 & 1.56 & 1.68 & 1.38 & 1.30 & 1.32 & 1.43 & 1.36 \\
\hline $\mathrm{Na}$ & 0.11 & 0.08 & 0.10 & 0.51 & 0.55 & 0.48 & 0.36 & 0.42 \\
\hline Sum B site & 2.00 & 2.00 & 2.00 & 2.02 & 2.05 & 2.01 & 1.99 & 2.00 \\
\hline $\mathrm{Na}$ & 0.11 & 0.08 & 0.10 & 0.03 & 0.05 & 0.02 & 0.00 & 0.01 \\
\hline $\mathrm{K}$ & 0.07 & 0.06 & 0.07 & 0.11 & 0.09 & 0.04 & 0.03 & 0.07 \\
\hline Sum A site & 0.19 & 0.14 & 0.17 & 0.14 & 0.14 & 0.06 & 0.04 & 0.08 \\
\hline Pkbar (Hollister et al., 1987) & 3.12 & 4.52 & 4.87 & 3.55 & 3.65 & 3.43 & 1.12 & 1.57 \\
\hline Pkbar (Schmidt, 1992) & 3.64 & 4.82 & 5.12 & 4.00 & 4.09 & 3.90 & 1.21 & 2.34 \\
\hline
\end{tabular}


Mogahed, M. M.

Table 2: Electron microprobe analyses of the biotite from the granitic rock of Wadi El-Akhder area, southwestern Sinai Peninsula, Egypt.

\begin{tabular}{|c|c|c|c|c|c|c|c|c|}
\hline \multirow{2}{*}{$\begin{array}{l}\text { Rock type } \\
\text { Petro. Name }\end{array}$} & \multicolumn{8}{|c|}{$\begin{array}{c}\text { Syn-tectonic granitoids } \\
\text { Wadi El-Akhder granitic rocks (AGR) }\end{array}$} \\
\hline & \multicolumn{3}{|c|}{ Tonalites } & \multicolumn{5}{|c|}{ Granodiorites } \\
\hline Sample No. & \multicolumn{3}{|c|}{$\mathrm{T} 17 / 1$} & \multicolumn{5}{|c|}{ G19/1 } \\
\hline $\mathrm{SiO}_{2}$ & 41.58 & 40.78 & 40.44 & 38.81 & 38.55 & 37.91 & 38.82 & 38.55 \\
\hline $\mathrm{TiO}_{2}$ & 3.54 & 3.91 & 4.73 & 2.03 & 1.91 & 1.45 & 2.06 & 1.97 \\
\hline $\mathrm{Al}_{2} \mathrm{O}_{3}$ & 13.91 & 14.49 & 15.22 & 16.71 & 16.48 & 16.34 & 16.76 & 16.57 \\
\hline $\mathrm{FeO}^{*}$ & 20.11 & 21.37 & 20.51 & 24.57 & 24.41 & 25.98 & 24.91 & 23.49 \\
\hline $\mathrm{MnO}$ & 0.19 & 0.19 & 0.18 & 0.68 & 0.21 & 0.17 & 0.18 & 0.36 \\
\hline $\mathrm{MgO}$ & 10.73 & 9.48 & 8.65 & 7.45 & 8.06 & 7.51 & 8.14 & 8.41 \\
\hline $\mathrm{Na}_{2} \mathrm{O}$ & 0.12 & 0.17 & 0.16 & 0.31 & 0.11 & 0.16 & 0.12 & 0.19 \\
\hline $\mathrm{K}_{2} \mathrm{O}$ & 8.54 & 8.47 & 8.81 & 8.33 & 8.73 & 7.06 & 7.63 & 8.44 \\
\hline Total & 98.72 & 98.86 & 98.70 & 98.89 & 98.46 & 96.58 & 98.62 & 97.98 \\
\hline \multicolumn{9}{|c|}{ Formula based on 22 oxygen atoms } \\
\hline $\mathrm{Si}$ & 6.17 & 6.08 & 6.03 & 5.90 & 5.89 & 5.90 & 5.89 & 5.89 \\
\hline $\mathrm{Al}^{(\text {iv) }}$ & 1.83 & 1.92 & 1.97 & 2.10 & 2.11 & 2.10 & 2.11 & 2.11 \\
\hline $\mathrm{Al}^{(\mathrm{vi})}$ & 0.60 & 0.63 & 0.71 & 0.89 & 0.85 & 0.90 & 0.88 & 0.87 \\
\hline $\mathrm{Ti}$ & 0.40 & 0.44 & 0.53 & 0.23 & 0.22 & 0.17 & 0.23 & 0.23 \\
\hline $\mathrm{Fe}^{2+}$ & 2.00 & 2.17 & 2.06 & 2.61 & 2.61 & 2.86 & 2.65 & 2.49 \\
\hline $\mathrm{Mn}$ & 0.02 & 0.02 & 0.02 & 0.09 & 0.03 & 0.02 & 0.02 & 0.05 \\
\hline $\mathrm{Mg}$ & 2.37 & 2.11 & 1.92 & 1.69 & 1.84 & 1.74 & 1.84 & 1.91 \\
\hline $\mathrm{Na}$ & 0.03 & 0.05 & 0.05 & 0.09 & 0.03 & 0.05 & 0.04 & 0.06 \\
\hline $\mathrm{K}$ & 1.62 & 1.61 & 1.68 & 1.62 & 1.70 & 1.40 & 1.48 & 1.64 \\
\hline $\mathrm{FeO} / \mathrm{MgO}$ & 1.69 & 2.03 & 2.13 & 2.97 & 2.73 & 3.11 & 2.75 & 2.51 \\
\hline
\end{tabular}

\section{Amphibole}

Amphiboles constitute the main mafic minerals in the studied tonalite and granodiorite. Results of the analyzed amphiboles together with atoms per formula unit calculated on the basis of 23 oxygens are given in Table 1. The $\mathrm{Fe}^{3+}$ and $\mathrm{Fe}^{2+}$ were calculated using the 13-CNK method (Leake et al. 1997; Leake et al. 2003). The analyzed amphiboles are mostly possess $\mathrm{Ti}$ (apfu) $>0.1$ (Table 1), a characteristic of primary igneous amphiboles (Girardeau and Mevel 1982). In addition, the analyzed amphiboles show decreasing of $\mathrm{Si}, \mathrm{Ti}, \mathrm{Al}, \mathrm{Fe}^{+3}$ and $\mathrm{Mg}$ from the tonalites to granodiorites, whereas the $\mathrm{Na}, \mathrm{K}$ and $\mathrm{Ca}$ increase.

According to the classification of (Leake et al. 2003), the analyzed amphiboles from the studied granitic rocks are mainly calcic in composition (Fig. 4a). The analyzed amphiboles are essentially actinolite in tonalites and granodiorite (Fig. 4b) and exhibiting a general increasing of their $\mathrm{Na}$ and $\mathrm{K}$ contents with the increasing of their Al contents (Table 1).



Fig. 4. (a) $\mathrm{B}_{\mathrm{Na}}$ vs. $\mathrm{B}_{(\mathrm{Ca}+\mathrm{Na})}$ classification diagram of amphibole (Leake et al. 1997). Symbol in Figs. 4-10: tonalites and $-\square$ granodiorites.

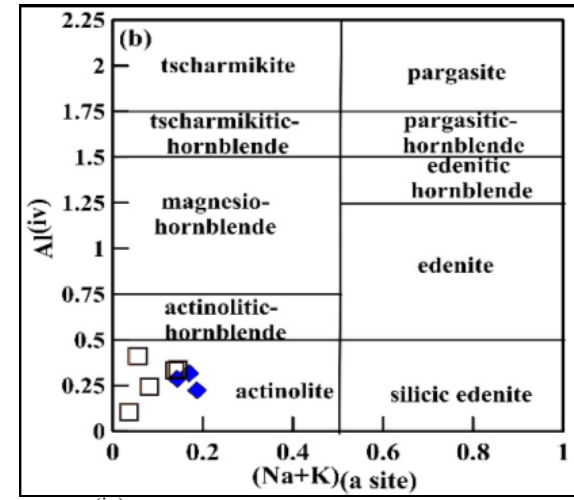

(b) Plot of $\mathrm{Al}^{\text {(iv) }}$ vs. $(\mathrm{Na}+\mathrm{K})$ in A-site for the analyzed amphiboles from the studied granitic rocks.

Nomenclature from (Hawthorne et al. 2012). 
Geochemistry and petrogenesis of the calc-alkaline Granitoids

Generally, the amphibole compositions could be used as pressure indicator as their $\mathrm{Al}$ (apfu) is general increase with pressure based on the equation $\left[\mathrm{P}(\mathrm{kbar})=5.64\left(\mathrm{Al}^{\mathrm{iv}}+\mathrm{Al}^{\mathrm{vi}}-4.76\right)\right]$ suggested by Hollister et al. (1987). The studied AGR have an average of Al (apfu) ( 1.39). Accordingly, the average of calculated crystallization pressure estimates of the AGR of about $3.23 \mathrm{Kbar}$ (Table. 1). In addition, the hornblende barometry of (Schmidt 1992) was applied for the studied granitic rocks and yield average pressure of about (3.64 \pm 0.6$)$ Kbar (Table 1).

\section{Biotite}

Biotite occurs in all the studied granitic samples. Results of EMPA are shown in Table (2) together with the number of atoms per formula unit based on 22 oxygens in the anhydrous total. Based on the chemical composition of the analyzed biotite they are belonging to primary magmatic biotites (Fig. 5a). Moreover, the biotite changes from calc-alkaline to slightly peraluminous in the AGR (Figs. $5 \mathrm{~b}$ and c). The analyzed biotite has $\mathrm{Al}_{2} \mathrm{O}_{3}$ contents with an average of 16.02 and has high contents of $\mathrm{FeO}, \mathrm{MgO}$ and $\mathrm{Na}_{2} \mathrm{O}$ and low contents of $\mathrm{K}_{2} \mathrm{O}$ (Table 2). On the $\mathrm{Fe}^{2+} /\left(\mathrm{Mg}+\mathrm{Fe}^{2+}\right)$ vs. $\mathrm{Al}$ (apfu) diagram of (Rieder et al. 1999), the studied mica from AGR lies within the chemical composition of biotite (Fig. 5d).

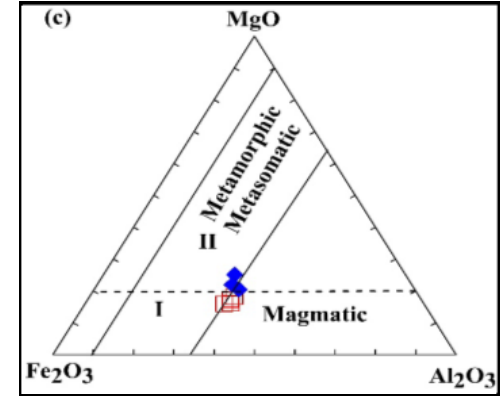

Fig. 5: a) $\mathrm{Fe}_{2} \mathrm{O}_{3}-\mathrm{MgO}-\mathrm{Al}_{2} \mathrm{O}_{3}$ ternary diagram of biotites in the studied granitic rocks. Dashed line separates biotites of magmatic environment from those of metamorphic-metasomatic environment (Gokhale 1968), whereas the area between the solid lines indicates the zone demarcated by Nockolds (1947) for igneous rocks.



(c) $\mathrm{Mg}_{(\mathrm{apfu})}-\mathrm{Al}_{(\mathrm{apfu})}$ diagram (Nachit et al. 1985) applied for the analyzed biotites.



(b) $\mathrm{FeO}^{*}$ vs. $\mathrm{Al}_{2} \mathrm{O}_{3}$ biotite discriminant diagram (Abdel-Rahman 1994) for the analyzed biotites.



(d) Nomenclature scheme of the studied biotite based on the $\mathrm{Al}$ vs. $\mathrm{Fe}^{2+} /\left(\mathrm{Mg}+\mathrm{Fe}^{2+}\right)$ diagram of Deer et al. (1992).

\section{Feldspars}

The plagioclase and alkali feldspars were analyzed from all the studied granitic types and their analyzed data are given in Tables 3 and 4.

Generally, the analyzed plagioclase from AGR are homogeneous in composition and possess high contents of $\mathrm{Al}_{2} \mathrm{O}_{3}$ (19.21-27.11 wt.\%), $\mathrm{Na}_{2} \mathrm{O}$ (7.41-12.07 wt.\%) and with low contents of $\mathrm{CaO}$ (3.23-8.61 wt.\%; Table 3). They are sodic enriched and have wide variation of anorthite contents $\left(\mathrm{An}_{12.35-37.98}\right)$ and ranging in composition from oligoclase to calcic-andesine (Fig. 6). In addition, the alkali feldspars from AGR (Fig. 4g) have composition ranging from $\left(\mathrm{An}_{0.66} \mathrm{Ab}_{15.48} \mathrm{Or}_{83.86}\right)$ to $\left(\mathrm{An}_{0.78} \mathrm{Ab}_{2.20} \mathrm{Or}_{97.02}\right)$. 
Mogahed, M. M.

Table 3: Electron microprobe analyses of the plagioclase feldspars from Wadi El-Akhder area, SW Sinai Peninsula.

\begin{tabular}{|c|c|c|c|c|c|c|c|c|c|}
\hline Rock type & \multicolumn{8}{|c|}{$\begin{array}{c}\text { Syn-tectonic granitoids } \\
\text { Wadi El-Akhder granitic rocks (AGR) }\end{array}$} & \\
\hline Petro. Name & \multicolumn{4}{|c|}{ Tonalites } & \multicolumn{5}{|c|}{ Granodiorites } \\
\hline Sample No. & \multicolumn{4}{|c|}{$\mathrm{T} 17 / 1$} & \multicolumn{5}{|c|}{ G19/1 } \\
\hline $\mathrm{SiO}_{2}$ & 60.98 & 61.12 & 59.34 & 60.37 & 63.41 & 62.55 & 57.14 & 58.19 & 56.59 \\
\hline $\mathrm{Al}_{2} \mathrm{O}_{3}$ & 24.41 & 24.03 & 25.51 & 24.17 & 20.11 & 19.21 & 25.63 & 24.26 & 27.11 \\
\hline $\mathrm{FeO}^{*}$ & 0.15 & 0.27 & 0 & 0.18 & 0.14 & 0.11 & 0.08 & 0.12 & 0 \\
\hline $\mathrm{CaO}$ & 5.61 & 5.61 & 7.06 & 6.04 & 4.52 & 3.23 & 6.07 & 8.61 & 7.55 \\
\hline $\mathrm{Na}_{2} \mathrm{O}$ & 8.03 & 8.15 & 7.41 & 8.13 & 10.11 & 12.07 & 8.48 & 7.64 & 8.05 \\
\hline $\mathrm{K}_{2} \mathrm{O}$ & 0.35 & 0.64 & 0.22 & 0.25 & 0.78 & 0.91 & 0.91 & 0.24 & 0.11 \\
\hline Total & 99.53 & 99.82 & 99.54 & 99.14 & 99.07 & 98.08 & 98.31 & 99.06 & 99.41 \\
\hline $\mathrm{CaO} /\left(\mathrm{Na}_{2} \mathrm{O}+\mathrm{K}_{2} \mathrm{O}\right)$ & 0.67 & 0.64 & 0.93 & 0.72 & 0.42 & 0.25 & 0.65 & 1.09 & 0.93 \\
\hline \multicolumn{10}{|c|}{ Cations calculated on basis of 32 oxygens } \\
\hline $\mathrm{Si}$ & 2.72 & 2.72 & 2.66 & 2.71 & 2.81 & 2.76 & 2.56 & 2.62 & 2.52 \\
\hline $\mathrm{Ti}$ & 0.00 & 0.00 & 0.00 & 0.00 & 0.00 & 0.00 & 0.00 & 0.00 & 0.00 \\
\hline$\overline{\mathrm{Al}}$ & 1.29 & 1.26 & 1.35 & 1.28 & 1.05 & 1.00 & 1.35 & 1.29 & 1.42 \\
\hline $\mathrm{Fe}^{3+}$ & 0.00 & 0.01 & 0.00 & 0.01 & 0.01 & 0.00 & 0.00 & 0.00 & 0.00 \\
\hline $\mathrm{Fe}^{2+}$ & 0.01 & 0.00 & 0.00 & 0.00 & 0.00 & 0.00 & 0.00 & 0.00 & 0.00 \\
\hline $\mathrm{Mn}$ & 0.00 & 0.00 & 0.00 & 0.00 & 0.00 & 0.00 & 0.00 & 0.00 & 0.00 \\
\hline $\mathrm{Mg}$ & 0.00 & 0.00 & 0.00 & 0.00 & 0.00 & 0.00 & 0.00 & 0.00 & 0.00 \\
\hline $\mathrm{Ca}$ & 0.27 & 0.27 & 0.34 & 0.29 & 0.21 & 0.15 & 0.29 & 0.41 & 0.36 \\
\hline $\mathrm{Ba}$ & 0.00 & 0.00 & 0.00 & 0.00 & 0.00 & 0.00 & 0.00 & 0.00 & 0.00 \\
\hline $\mathrm{Na}$ & 0.70 & 0.70 & 0.64 & 0.71 & 0.87 & 1.03 & 0.74 & 0.67 & 0.69 \\
\hline$\overline{\mathrm{K}}$ & 0.02 & 0.04 & 0.01 & 0.01 & 0.04 & 0.05 & 0.05 & 0.01 & 0.01 \\
\hline tot. cat. & 5.00 & 5.00 & 5.00 & 5.00 & 5.00 & 5.00 & 5.00 & 5.00 & 5.00 \\
\hline An & 27.29 & 26.56 & 34.05 & 28.69 & 19.04 & 12.35 & 26.98 & 37.89 & 33.93 \\
\hline$\overline{\mathrm{Ab}}$ & 70.68 & 69.83 & 64.68 & 69.89 & 77.05 & 83.51 & 68.21 & 60.85 & 65.48 \\
\hline Or & 2.03 & 3.61 & 1.26 & 1.41 & 3.91 & 4.14 & 4.82 & 1.26 & 0.59 \\
\hline
\end{tabular}

Table 4: Electron microprobe analyses of the alkali-feldspars from Wadi El-Akhder area, SW Sinai.

\begin{tabular}{|c|c|c|c|c|c|c|}
\hline \multirow{2}{*}{$\begin{array}{l}\text { Rock type } \\
\text { Petro. Name } \\
\end{array}$} & \multicolumn{6}{|c|}{$\begin{array}{c}\text { Syn-tectonic granitoids } \\
\text { Wadi El-Akhder granitic rocks (AGR) }\end{array}$} \\
\hline & \multicolumn{3}{|c|}{ Tonalites } & \multicolumn{3}{|c|}{ Granodiorites } \\
\hline Sample No. & \multicolumn{3}{|c|}{$\mathrm{T} 17 / 1$} & \multicolumn{3}{|c|}{ G19/1 } \\
\hline $\mathrm{SiO}_{2}$ & 63.14 & 63.37 & 63.01 & 63.18 & 63.71 & 63.55 \\
\hline $\mathrm{Al}_{2} \mathrm{O}_{3}$ & 18.71 & 18.34 & 18.31 & 18.55 & 18.63 & 18.34 \\
\hline $\mathrm{FeO}^{*}$ & 0.39 & 0.13 & 0.11 & 0.31 & 0.00 & 0.31 \\
\hline $\mathrm{CaO}$ & 0.26 & 0.15 & 0.12 & 0.23 & 0.11 & 0.16 \\
\hline $\mathrm{Na}_{2} \mathrm{O}$ & 0.56 & 0.56 & 0.59 & 0.31 & 0.51 & 0.25 \\
\hline $\mathrm{K}_{2} \mathrm{O}$ & 16.31 & 16.21 & 16.67 & 16.51 & 16.31 & 16.73 \\
\hline Total & 99.37 & 98.76 & 98.81 & 99.09 & 99.27 & 99.34 \\
\hline \multicolumn{7}{|c|}{ Cations calculated on basis of 32 oxygens } \\
\hline $\mathrm{Si}$ & 2.93 & 2.96 & 2.94 & 2.95 & 2.96 & 2.96 \\
\hline $\mathrm{Ti}$ & 0.00 & 0.00 & 0.00 & 0.00 & 0.00 & 0.00 \\
\hline$\overline{\mathrm{Al}}$ & 1.02 & 1.01 & 1.01 & 1.02 & 1.02 & 1.01 \\
\hline $\mathrm{Fe} 3$ & 0.02 & 0.01 & 0.00 & 0.01 & 0.00 & 0.01 \\
\hline $\mathrm{Fe} 2$ & 0.00 & 0.00 & 0.00 & 0.00 & 0.00 & 0.00 \\
\hline $\mathrm{Mn}$ & 0.00 & 0.00 & 0.00 & 0.00 & 0.00 & 0.00 \\
\hline$\overline{\mathrm{Mg}}$ & 0.00 & 0.00 & 0.00 & 0.00 & 0.00 & 0.00 \\
\hline $\mathrm{Ca}$ & 0.01 & 0.01 & 0.01 & 0.01 & 0.01 & 0.01 \\
\hline $\mathrm{Ba}$ & 0.00 & 0.00 & 0.00 & 0.00 & 0.00 & 0.00 \\
\hline $\mathrm{Na}$ & 0.05 & 0.05 & 0.05 & 0.03 & 0.05 & 0.02 \\
\hline $\mathrm{K}$ & 0.97 & 0.97 & 0.99 & 0.98 & 0.97 & 0.99 \\
\hline Tot. cat. & 5.00 & 5.00 & 5.00 & 5.00 & 5.00 & 5.00 \\
\hline \multicolumn{7}{|c|}{ End members } \\
\hline$\overline{A n}$ & 1.26 & 0.73 & 0.57 & 1.12 & 0.54 & 0.78 \\
\hline$\overline{\mathrm{Ab}}$ & 4.90 & 4.95 & 5.08 & 2.74 & 4.51 & 2.20 \\
\hline Or & 93.85 & 94.32 & 94.35 & 96.13 & 94.95 & 97.02 \\
\hline
\end{tabular}


Geochemistry and petrogenesis of the calc-alkaline Granitoids

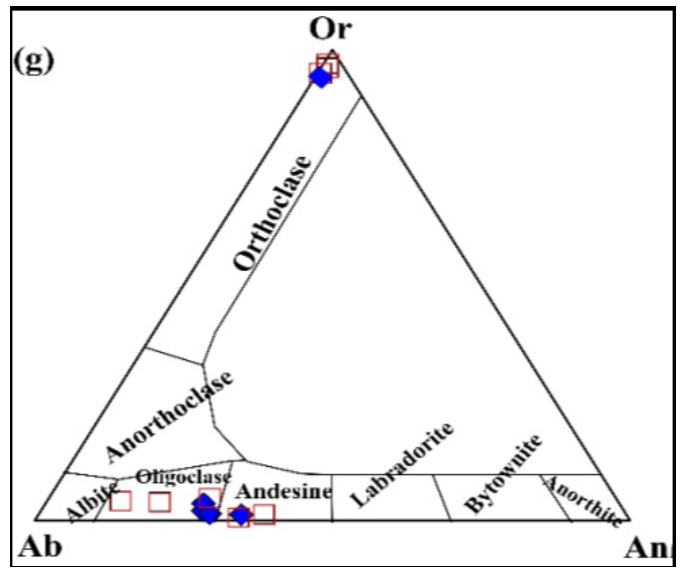

Fig. 6: Classification diagram for feldspars from the studied granitic rocks, compositional fields are after (Deer et al., 1992).

\section{MODAL ANALYSIS}

The modal abundances of the quartz and feldspars for the studied granitic rocks are given in Table 5 . The modal proportions of alkali feldspar, plagioclase and quartz are shown in figure 7. Based on the IUGS nomenclature (Streckeisen 1976), the studied AGR samples plot in the field of tonalite and granodiorites.

Table 5: Modal analyses for the granitic rocks from Wadi El-Akhder area, SW Sinai Peninsula.

\begin{tabular}{|c|c|c|c|c|c|c|c|c|c|c|}
\hline Rock type & \multicolumn{10}{|c|}{$\begin{array}{l}\text { Syn-tectonic granitoids } \\
\text { Wadi El-Akhder granitic rocks (AGR) }\end{array}$} \\
\hline Petro. Name & \multicolumn{4}{|c|}{ Tonalites } & \multicolumn{6}{|c|}{ Granodiorites } \\
\hline \multicolumn{11}{|c|}{ Modal components } \\
\hline Quartz & 27.31 & 27.16 & 28.50 & 24.78 & 21.94 & 24.30 & 24.38 & 25.09 & 23.26 & 28.76 \\
\hline Orthoclase & 8.92 & 7.92 & 6.62 & 14.24 & 15.25 & 11.41 & 12.29 & 10.76 & 16.78 & 16.13 \\
\hline Albite & 28.01 & 27.84 & 28.52 & 29.95 & 35.03 & 36.64 & 35.71 & 37.91 & 37.91 & 27.59 \\
\hline Anorthite & 20.95 & 21.74 & 21.13 & 15.10 & 12.92 & 13.43 & 12.47 & 11.73 & 7.14 & 16.32 \\
\hline Plagioclase & 48.95 & 49.58 & 49.65 & 45.06 & 47.95 & 50.07 & 48.18 & 49.64 & 45.05 & 43.90 \\
\hline
\end{tabular}



\section{WHOLE-ROCK CHEMISTRY}

The chemical analyses of the studied granitic rocks and some of their calculated normative mineral compositions are listed in Table 6 . Generally, the $\mathrm{SiO}_{2}$ contents are broadly high for granitic rocks. The studied granitic rocks exhibiting a range of $\mathrm{SiO}_{2}$ from approximately 64.98 to $66.08 \mathrm{wt} . \%$ for tonalites and 66.28 to 67.54 wt.\% for granodiorite (Table 6). Molar norm composition shown in a Q' vs. ANOR diagram indicate that the granitoids form a continuous suite from granodiorite to tonalite (Fig. 8).

Based on the classification diagram of the Egyptian granitoids (Hassan and Hashad 1990), the investigated tonalite and granodiorite are akin to the calc-alkaline Egyptian older granitoids (Fig. 9a). The TAS diagram of Le Maitre et al. (1989) was chosen among recent schemes as it provides a classification with alkaline and calc-alkaline fields (Fig. 9b). The dividing line between alkaline and non-alkaline series from Irvine and Baragar (1971) and Miyashiro (1978) have also been plotted for comparison. Almost all of the tonalites and granodiorites samples from the studied AGR plot in the calc-alkaline field (Fig. 9b). 


\section{Mogahed, M. M.}

The studied granitic rocks have relatively higher contents of $\mathrm{K}_{2} \mathrm{O}$ ranging from 1.12 to $2.84 \mathrm{wt} . \%$. Based on the nomenclature of Rickwood (1989), the AGR are classified as medium-K calc-alkaline rocks (Fig. 10a). Based on the aluminum saturation index $\left[\mathrm{A} / \mathrm{CNK}\right.$; molecular $\left.\mathrm{Al}_{2} \mathrm{O}_{3} /\left(\mathrm{CaO}+\mathrm{Na}_{2} \mathrm{O}+\mathrm{K}_{2} \mathrm{O}\right)\right]$ (Fig. $10 \mathrm{~b}$ ), the studied granitic rocks plot mainly in the metaluminous field. There is no significant gap between the compositionally different studied AGR types suggesting that the rocks of each series are cogenetic.



Fig. 8: $\mathrm{Q}^{\prime}[100 * \mathrm{Q} /(\mathrm{Q}+\mathrm{Or}+\mathrm{Ab}+\mathrm{An})]$ vs. $\mathrm{ANOR}$ $[100 \times \mathrm{An} /(\mathrm{Or}+\mathrm{An})]$ diagram for normative classification of the studied granitic rocks (Streckeisen and Le Maitre 1979).

Table 6: Whole-rock analyses for the granitic rocks from Wadi El-Akhder area, SW Sinai Peninsula.

\begin{tabular}{|c|c|c|c|c|c|c|c|c|c|c|}
\hline Rock type & \multicolumn{10}{|c|}{$\begin{array}{l}\text { Syn-tectonic granitoids } \\
\text { Wadi El-Akhder granitic rocks (AGR) }\end{array}$} \\
\hline Petro. Name & \multicolumn{4}{|c|}{ Tonalites } & \multicolumn{6}{|c|}{ Granodiorites } \\
\hline Sample & $\mathrm{T} 17 / 1$ & T26/1 & T28/2 & T29/1 & G19/1 & $\mathrm{G} 21 / 1$ & G24/1 & G39/2 & $\mathrm{G} / 41 / 1$ & G64/1 \\
\hline Lat. $28^{\circ} \mathrm{N}$ & 4652 & $46^{\prime} 22^{\prime \prime}$ & $46^{\prime} 6^{\prime \prime}$ & $48^{\prime} 7^{\prime \prime}$ & $48^{\prime} 48^{\prime \prime}$ & $45^{\prime} 4^{\prime \prime}$ & $43^{\prime} 30^{\prime \prime}$ & $43^{`} 57^{\prime \prime}$ & $46^{\prime} 22^{\prime \prime}$ & $47^{`} 7^{`}$ \\
\hline Long. $33^{\circ} \mathrm{E}$ & $45^{\prime} 2^{\prime \prime}$ & $47^{\prime} 3$ & $48^{\prime} 11^{\prime \prime}$ & $50^{\prime} 17^{\prime \prime}$ & $42^{\prime} 42^{\prime \prime}$ & $42^{\prime} 15^{\prime}$ & $44^{\prime \prime}$ & $4642^{\prime \prime}$ & $50 ` 39^{\prime \prime}$ & $523^{\prime \prime}$ \\
\hline $\mathrm{SiO}_{2}$ & 64.98 & 65.17 & 65.53 & 66.08 & 66.28 & 66.99 & 66.26 & 66.90 & 67.36 & 67.54 \\
\hline $\mathrm{TiO}_{2}$ & 0.91 & 0.86 & 0.82 & 0.77 & 0.67 & 0.66 & 0.61 & 0.59 & 0.56 & 0.52 \\
\hline $\mathrm{Al}_{2} \mathrm{O}_{3}$ & 15.12 & 14.83 & 14.50 & 13.97 & 14.34 & 14.13 & 13.76 & 13.64 & 13.06 & 14.30 \\
\hline $\mathrm{FeO}^{*}$ & 3.92 & 3.45 & 3.77 & 4.26 & 4.70 & 3.92 & 3.21 & 3.68 & 3.69 & 2.87 \\
\hline $\mathrm{MnO}$ & 0.18 & 0.13 & 0.13 & 0.11 & 0.10 & 0.12 & 0.11 & 0.15 & 0.05 & 0.12 \\
\hline $\mathrm{MgO}$ & 2.41 & 2.86 & 2.21 & 2.17 & 1.81 & 1.74 & 1.51 & 1.48 & 1.27 & 1.16 \\
\hline $\mathrm{CaO}$ & 4.63 & 4.91 & 5.18 & 4.96 & 4.63 & 4.49 & 4.26 & 3.96 & 3.67 & 4.05 \\
\hline $\mathrm{Na}_{2} \mathrm{O}$ & 3.31 & 3.29 & 3.37 & 3.54 & 4.14 & 4.33 & 4.22 & 4.48 & 4.48 & 3.26 \\
\hline $\mathrm{K}_{2} \mathrm{O}$ & 1.51 & 1.34 & 1.12 & 2.41 & 2.58 & 1.93 & 2.08 & 1.82 & 2.84 & 2.73 \\
\hline $\mathrm{P}_{2} \mathrm{O}_{5}$ & 0.31 & 0.34 & 0.19 & 0.23 & 0.05 & 0.13 & 0.10 & 0.13 & 0.13 & 0.16 \\
\hline LOI & 0.73 & 0.98 & 1.42 & 1.03 & 0.63 & 1.24 & 2.63 & 2.66 & 2.97 & 2.54 \\
\hline Total & 99.14 & 99.63 & 98.92 & 99.21 & 99.32 & 99.53 & 98.97 & 98.54 & 98.93 & 98.71 \\
\hline \multicolumn{11}{|c|}{ Trace and REE elements contents (ppm) } \\
\hline $\mathrm{V}$ & 48 & 82 & 72 & 61 & 97 & 83 & 69 & 116 & 78 & 78 \\
\hline $\mathrm{Cr}$ & 51 & 57 & 48 & 43 & 40 & 38 & 40 & 36 & 40 & 41 \\
\hline $\mathrm{Co}$ & 16 & 18 & 14 & 18 & 13 & 14 & 21 & 8 & 6 & 10 \\
\hline $\mathrm{Ni}$ & 40 & 22 & 32 & 28 & 18 & 14 & 14 & 14 & 12 & 19 \\
\hline $\mathrm{Cu}$ & 9 & 7 & 6 & 8 & 8 & 9 & 761 & 3 & 9 & 7 \\
\hline $\mathrm{Zn}$ & 11 & 42 & 13 & 27 & 30 & 24 & 53 & 12 & 25 & 40 \\
\hline $\mathrm{Cs}$ & 5 & 5 & 4 & 4 & 3 & 5 & 2 & 2 & 2 & 3 \\
\hline $\mathrm{Ga}$ & 12 & 12 & 12 & 18 & 17 & 16 & 15 & 16 & 15 & 20 \\
\hline $\mathrm{Rb}$ & 93 & 86 & 77 & 92 & 112 & 88 & 102 & 117 & 102 & 117 \\
\hline $\mathrm{Sr}$ & 805 & 770 & 865 & 835 & 519 & 593 & 454 & 398 & 326 & 326 \\
\hline $\mathrm{Y}$ & 6 & 7 & 8 & 9 & 7 & 7 & 7 & 6 & 6 & 5 \\
\hline $\mathrm{Zr}$ & 149 & 104 & 151 & 140 & 138 & 93 & 157 & 147 & 141 & 137 \\
\hline $\mathrm{Nb}$ & 12 & 11 & 15 & 15 & 11 & 10 & 17 & 16 & 13 & 13 \\
\hline $\mathrm{Ba}$ & 1221 & 1296 & 1430 & 1675 & 1199 & 1284 & 1056 & 1140 & 1268 & 996 \\
\hline $\mathrm{Hf}$ & 4 & 3 & 3 & 2 & 3 & 3 & 4 & 4 & 4 & 5 \\
\hline $\mathrm{Ta}$ & 1 & 2 & 2 & 2 & 1 & 2 & 2 & 1 & 2 & 2 \\
\hline $\mathrm{Pb}$ & 21 & 29 & 19 & 18 & 17 & 27 & 23 & 21 & 25 & 25 \\
\hline
\end{tabular}

Table 6. (continued) 
Geochemistry and petrogenesis of the calc-alkaline Granitoids

\begin{tabular}{|c|c|c|c|c|c|c|c|c|c|c|}
\hline \multirow{3}{*}{$\begin{array}{l}\text { Rock type } \\
\text { Petro. Name } \\
\text { Sample } \\
\end{array}$} & \multicolumn{10}{|c|}{$\begin{array}{l}\text { Syn-tectonic granitoids } \\
\text { Wadi El-Akhder granitic rocks (AGR) }\end{array}$} \\
\hline & \multicolumn{4}{|c|}{ Tonalites } & \multicolumn{6}{|c|}{ Granodiorites } \\
\hline & $\mathrm{T} 17 / 1$ & T26/1 & $\mathrm{T} 28 / 2$ & T29/1 & G19/1 & G21/1 & G24/1 & G39/2 & $\mathrm{G} / 41 / 1$ & G64/1 \\
\hline Th & 14.48 & 16.48 & 15.48 & 16.09 & 16.02 & 16.56 & 22.08 & 24.26 & 27.04 & 25.87 \\
\hline $\mathrm{U}$ & 8.50 & 4.70 & 5.04 & 4.40 & 7.50 & 5.30 & 5.70 & 6.03 & 6.60 & 5.80 \\
\hline $\mathrm{La}$ & 25.90 & 32.17 & 38.19 & 42.56 & 30.75 & 35.74 & 41.47 & 46.21 & 51.42 & 58.52 \\
\hline $\mathrm{Ce}$ & 47.48 & 55.18 & 62.80 & 69.99 & 56.45 & 62.80 & 69.88 & 76.13 & 102.84 & 84.84 \\
\hline $\operatorname{Pr}$ & 4.98 & 5.67 & 6.45 & 7.34 & 6.06 & 6.60 & 8.01 & 8.91 & 11.29 & 12.56 \\
\hline $\mathrm{Nd}$ & 15.95 & 18.15 & 21.10 & 24.52 & 17.46 & 22.11 & 25.66 & 29.82 & 36.98 & 25.66 \\
\hline $\mathrm{Sm}$ & 3.82 & 4.44 & 4.84 & 5.75 & 5.07 & 5.07 & 6.57 & 7.47 & 8.86 & 9.88 \\
\hline $\mathrm{Eu}$ & 1.20 & 1.45 & 1.28 & 1.69 & 1.18 & 2.11 & 1.70 & 1.73 & 2.02 & 1.77 \\
\hline $\mathrm{Gd}$ & 2.63 & 3.20 & 2.87 & 3.64 & 3.09 & 5.63 & 4.08 & 4.84 & 5.75 & 6.68 \\
\hline $\mathrm{Tb}$ & 0.40 & 0.46 & 0.52 & 0.59 & 0.49 & 0.61 & 0.67 & 0.86 & 1.04 & 0.79 \\
\hline Dy & 2.20 & 2.68 & 3.05 & 3.47 & 2.14 & 3.74 & 3.36 & 4.07 & 4.84 & 6.25 \\
\hline Ho & 0.39 & 0.47 & 0.35 & 0.59 & 0.46 & 0.66 & 0.71 & 0.81 & 0.96 & 0.74 \\
\hline Er & 0.95 & 1.32 & 1.13 & 1.56 & 1.28 & 1.58 & 1.92 & 2.23 & 2.48 & 2.71 \\
\hline $\mathrm{Tm}$ & 0.13 & 0.18 & 0.19 & 0.22 & 0.19 & 0.26 & 0.26 & 0.30 & 0.36 & 0.29 \\
\hline $\mathrm{Yb}$ & 0.82 & 1.04 & 1.48 & 1.22 & 1.31 & 1.56 & 2.06 & 2.29 & 2.55 & 2.06 \\
\hline $\mathrm{Lu}$ & 0.13 & 0.16 & 0.24 & 0.20 & 0.22 & 0.27 & 0.30 & 0.35 & 0.37 & 0.41 \\
\hline$\sum \mathrm{REE}$ & 107 & 127 & 144 & 163 & 126 & 149 & 167 & 186 & 232 & 213 \\
\hline \multicolumn{11}{|c|}{ Petrochemical parameters } \\
\hline $\mathrm{Q}^{\prime}$ & 32.06 & 32.08 & 33.62 & 29.47 & 25.78 & 28.43 & 23.63 & 25.08 & 22.67 & 24.84 \\
\hline ANQR & 70.13 & 73.30 & 76.15 & 51.47 & 45.86 & 52.90 & 65.16 & 63.08 & 67.02 & 48.91 \\
\hline $\mathrm{Cao} / \mathrm{Na}_{2} \mathrm{O}$ & 1.40 & 1.49 & 1.54 & 1.40 & 1.12 & 1.04 & 1.01 & 0.88 & 0.82 & 1.24 \\
\hline $\mathrm{Al}_{2} \mathrm{O}_{3} / \mathrm{TiO}_{2}$ & 16.69 & 17.33 & 17.60 & 18.05 & 21.44 & 21.43 & 22.59 & 23.08 & 23.37 & 27.61 \\
\hline $\mathrm{Fe}_{2} \mathrm{O}_{3} / \mathrm{MgO}$ & 2.10 & 1.72 & 2.01 & 1.82 & 2.26 & 2.17 & 2.26 & 1.85 & 2.01 & 2.00 \\
\hline $\mathrm{A} / \mathrm{CNK}$ & 0.98 & 0.94 & 0.90 & 0.80 & 0.80 & 0.99 & 0.94 & 0.96 & 0.96 & 0.99 \\
\hline AI & 0.47 & 0.46 & 0.47 & 0.60 & 0.67 & 0.60 & 0.54 & 0.54 & 0.54 & 0.63 \\
\hline$(\mathrm{Ga} / \mathrm{Al}) * 10^{4}$ & 1.89 & 1.98 & 1.82 & 1.95 & 2.01 & 2.12 & 2.20 & 2.14 & 2.12 & 2.28 \\
\hline $\mathrm{Rb} / \mathrm{Sr}$ & 0.12 & 0.11 & 0.09 & 0.11 & 0.22 & 0.15 & 0.23 & 0.29 & 0.31 & 0.36 \\
\hline $\mathrm{Ba} / \mathrm{Sr}$ & 1.52 & 1.68 & 1.65 & 2.01 & 2.31 & 2.16 & 2.32 & 2.87 & 3.89 & 3.06 \\
\hline $\mathrm{Ba} / \mathrm{Rb}$ & 13.07 & 15.01 & 18.62 & 18.17 & 10.69 & 14.67 & 10.31 & 9.77 & 12.38 & 8.54 \\
\hline $\mathrm{Th} / \mathrm{La}$ & 0.40 & 0.43 & 0.36 & 0.36 & 0.52 & 0.46 & 0.53 & 0.52 & 0.53 & 0.44 \\
\hline $\mathrm{Th} / \mathrm{Ta}$ & 10.13 & 10.84 & 8.46 & 9.52 & 10.75 & 10.48 & 10.99 & 17.71 & 16.69 & 16.07 \\
\hline $\mathrm{La} / \mathrm{Nb}$ & 2.97 & 3.60 & 2.92 & 2.91 & 2.89 & 3.51 & 2.47 & 2.87 & 4.08 & 4.57 \\
\hline $\mathrm{Ba} / \mathrm{Nb}$ & 100.87 & 122.22 & 96.55 & 109.25 & 112.69 & 126.19 & 62.99 & 70.90 & 100.63 & 77.72 \\
\hline $\mathrm{Eu} / \mathrm{Eu}^{*}$ & 1.09 & 1.12 & 0.96 & 1.05 & 0.84 & 1.20 & 0.93 & 0.82 & 0.81 & 0.63 \\
\hline$(\mathrm{La} / \mathrm{Lu})_{\mathrm{N}}$ & 20.01 & 20.94 & 16.48 & 21.84 & 14.30 & 13.68 & 14.27 & 13.68 & 14.27 & 14.91 \\
\hline
\end{tabular}

Lat., latitude (all locations start with $28^{\circ} \mathrm{N}$ ); Long., longitude (all locations start with $33^{\circ} \mathrm{E}$ ); $\mathrm{FeO} *$ refers total $\mathrm{Fe}$ is expressed as $\mathrm{FeO} ;(\mathrm{AI}=(\mathrm{Na}+\mathrm{K}) / \mathrm{Al}($ atom \%) is the calculated agpaitic index.



Fig. 9a: Classification diagram of the Egyptian granitic rocks after (Hassan and Hashad 1990). The trondhjemitic (TR) and calc-alkaline (CA) trends are from Barker and Arth (1976).



Fig. 9 b: Total alkalis $\left(\mathrm{Na}_{2} \mathrm{O}+\mathrm{K}_{2} \mathrm{O}\right)$ vs. silica $\left(\mathrm{SiO}_{2}\right)$ diagram (Le Maitre et al. 1989). Dashed lines of Irvine and Baragar (1971) and Miyashiro (1978) are lines discriminate between alkaline and subalkaline rocks. 
Mogahed, M. M.



Fig. 10a: $\mathrm{K}_{2} \mathrm{O}$ vs. $\mathrm{SiO}_{2}$ variation diagram for the studied granitic rocks. Fields are after (Rickwood 1989).



b: $\mathrm{Al} /(\mathrm{Na}+\mathrm{K})$ vs. $\mathrm{Al} /(\mathrm{Ca}+\mathrm{Na}+\mathrm{K})(\mathrm{mol} . \%)$ : fields are after (Maniar and Piccoli 1989).

Harker variation diagrams were used to evaluate major oxides and trace elements variations in the studied granitic rocks. The studied AGR have high contents of $\mathrm{Al}_{2} \mathrm{O}_{3}, \mathrm{TiO}_{2}, \mathrm{CaO}$ and $\mathrm{Fe}_{2} \mathrm{O}_{3}$ (Fig. 11). Most elements of the studied AGR show linear decreases with increasing $\mathrm{SiO}_{2}$; except $\mathrm{K}_{2} \mathrm{O}, \mathrm{Na}_{2} \mathrm{O}, \mathrm{Rb}$, $\mathrm{Nb}, \mathrm{Zr}$ and $\mathrm{Ba}$ (Table 6; Fig. 11), which probably indicate fractionation of plagioclase $(\mathrm{CaO}$ and $\mathrm{Sr}$ decrease), and amphibole $\left(\mathrm{CaO}, \mathrm{Fe}_{2} \mathrm{O}_{3}, \mathrm{MgO}, \mathrm{TiO}_{2}\right.$ and $\mathrm{Y}$ decrease). The contents of both $\mathrm{K}_{2} \mathrm{O}$ and $\mathrm{Ba}$ show an initial increase until 66 wt. $\% \mathrm{SiO}_{2}$ followed by a decrease in the higher $\mathrm{SiO}_{2}$ range, suggesting the initiation of K-feldspar fractionation (Fig. 11).

Fig. 11: Harker variation diagrams for the studied granites. Arrows indicate theoretical Rayleigh fractionation vectors modeled for crystallization of individual mineral phases. Partition coefficients used for the modeling are from the compilation of Rollinson (1993). Abbreviations: amp = amphibole; $\mathrm{plg}=$ plagioclase $; \mathrm{K}$-feld $=\mathrm{K}$-feldspar; bio $=$ biotite $; \mathrm{cpx}=$ clinopyroxene; $\mathrm{opx}=$ orthopyroxene.




Geochemistry and petrogenesis of the calc-alkaline Granitoids

The near-linear variations of major oxides with silica can be explained either by magmatic differentiation from a common parent melt, or by mixing of melts from two (or more) discrete compositional sources (e.g. mantle- and crust-derived melts), or both. Moreover, the studied granitic rocks have very high $\mathrm{FeO} / \mathrm{MgO}$ ratios $(>1.72$, Table 6$)$, indicating that their parental magmas have experienced extensive magmatic differentiation (e.g., Whalen et al. 1987). The studied granitic rocks have a wide range of $\mathrm{CaO} / \mathrm{Na}_{2} \mathrm{O}$ ratios being decrease from tonalites to granodiorites (Table 6) and their $\mathrm{Al}_{2} \mathrm{O}_{3} / \mathrm{TiO}_{2}$ ratios have a negative correlation with $\mathrm{CaO} / \mathrm{Na}_{2} \mathrm{O}$ ratios (Fig. 12).



Fig. 12: Variation diagrams for the studied granites. a) $\mathrm{CaO} / \mathrm{Na}_{2} \mathrm{O}$ versus $\mathrm{Al}_{2} \mathrm{O}_{3} / \mathrm{TiO}_{2}$ (Sylvester 1998). The line of the mixing of basaltic and granitic melts was calculated between Phanerozoic basalt (Condie 1993) (point M "mafic") and melt produced from crustal melt is taken as the $850^{\circ} \mathrm{C}, 10 \mathrm{kbar}$ crust-derived (experimental) melt (Point F "Felsic") after Patiño Douce and Johnston (1991). b) $\mathrm{Rb} / \mathrm{Ba}$ vs. $\mathrm{Rb} / \mathrm{Sr}$ diagram. End-member compositions for mafic and felsic melt are the same as in Fig. 12a. c) and (d) Variations of $\mathrm{CaO} / \mathrm{Na}_{2} \mathrm{O}$ and $\mathrm{Rb} / \mathrm{Sr}$, respectively with silica contents of the studied granitic rocks. e) $\left(\mathrm{FeO} *+\mathrm{MgO}+\mathrm{TiO}_{2}\right)$ versus $\mathrm{SiO}_{2}$ diagram. The line of the mixing of basaltic and granitic melts is the same as in Figure 12a. f) $\mathrm{Rb} / \mathrm{Ba}-\mathrm{Rb} / \mathrm{Sr}$. Dashed line separates the fields of the sources enriched and depleted in clay components (Sylvester 1998).

The concentrations of some incompatible trace elements (e.g., Ba, Sr, and Y) markedly decrease with increasing silica (from tonalites to granodiorites). The geochemical data of the studied granitic rocks shows varying ratios of incompatible elements with low values of $\mathrm{Rb} / \mathrm{Sr}$ ratio (0.09 to 0.36$)$, low values of $\mathrm{Ba} / \mathrm{Sr}$ ratio ranges from 0.92 to 1.73 and high values of $\mathrm{Ba} / \mathrm{Rb}$ ratio (8.54 to 18.62 ; Table 6). Such variation in these geochemical ratios (e.g., $\mathrm{Ba} / \mathrm{Rb}, \mathrm{Ba} / \mathrm{Sr}, \mathrm{Rb} / \mathrm{Sr}$ and $\mathrm{Rb} / \mathrm{Ba}$ ), indicates high fractionation nature of the studied granitic rocks, which can be attained by magmatic differentiation (Obiora and Ukaegbu 2010; Rajesh 2007; Taylor 1965). The limited variation in the ratios of the incompatible elements of $\mathrm{Rb} / \mathrm{Sr}, \mathrm{Ba} / \mathrm{Sr}$ and $\mathrm{Ba} / \mathrm{Rb}$ for each group of the studied granitic rocks (Table 6) indicates that granitic rock suite was affected by variable degree of partial melting (e.g., Guffanti et al. 1996; Roberts and Clemens 1993).

The values of High Field Strength Elements (HFSE) (U, Zr, Nb, Hf, Ta) and Large Ion Lithophile Elements (LILE) (Cs, $\mathrm{Ba}, \mathrm{Pb}, \mathrm{Sr}$, and $\mathrm{REE}$ ) of the studied granitic rocks (Table 6) compared well with the values of average granite and crust of Taylor and McLennan (1985). The enrichment of some of the HFSE like $\mathrm{Nb}$, Hf and $\mathrm{Ta}$ in the studied granitic rocks suggests volatile concentrations during the evolution of granites. The contents $\mathrm{Rb}$ and $\mathrm{Th}$ of the studied AGR (Table 6) show abnormally high values compared to average granite and crust of Taylor and McLennan (1995).

Rare-Earth Elements (REE) concentrations together with statistical parameters are listed in Table 6. The values of High Field Strength Elements (HFSE) (U, Zr, Nb, Hf, Ta) and Large Ion Lithophile 


\section{Mogahed, M. M.}

Elements (LILE) (Cs, Ba, $\mathrm{Pb}, \mathrm{Sr}$, and REE) of the studied granitic rocks compared well with the values of average granite and crust of Taylor and McLennan (1985). The enrichment of some of the HFSE like Nb, $\mathrm{Hf}$ and $\mathrm{Ta}$ in the studied granitic rocks suggests volatile concentrations during the evolution of granites. The contents $\mathrm{Rb}$ and $\mathrm{Th}$ of the studied AGR (Table 6) show abnormally high values compared to average granite and crust of Taylor and McLennan (1995).

The chondrite-normalized REE patterns are presented in Figure (13). All analyzed samples are characterized by fractionation between Light Rare-Earth Elements (LREE) and Heavy Rare-Earth Elements (HREE) (Figs. 13a and b). The high level of enrichment in the LREE relative to the HREE in all the studied granitic rocks suggests a high degree of fractionation. In addition, most of the analyzed samples of AGR are characterized by concave-upward REE profiles resulting from depletion of the middle REE (Gd to Er) relative to the other HREE (Fig. 13a). Moreover, the studied tonalite samples from AGR exhibit highly fractionated REE patterns $\left[(\mathrm{La} / \mathrm{Lu})_{\mathrm{N}}=16.48-21.84\right]$, whereas the studied granodiorite samples are less fractionated $\left[(\mathrm{La} / \mathrm{Lu})_{\mathrm{N}}=13.68-14.91\right]$. Generally, the studied AGR are characterized by moderately fractionated HREE segments, and have slight or no negative $\mathrm{Eu}$ anomalies $\left(\mathrm{Eu} / \mathrm{Eu}^{*}=0.63-\right.$ 1.20; Fig. 13a). Moreover, the REE concentrations of the studied AGR are conformable with those of calc-alkaline granitoids granites from the ANS (Fig. 13).

MORB-normalized element patterns for the studied granitic rocks (Fig. 13b) exhibiting enrichment in the most incompatible elements (e.g., $\mathrm{Rb}, \mathrm{Ba}$, and $\mathrm{Th}$ ) with respect to the LREE, and show decreasing of the enrichment factor with increasing compatibility of the elements (Fig. 13b). Moreover, the studied granitic rocks show an overall enrichment in large ion lithophile elements (LILE; Rb, Ba, Th, U, K) compared to normalization values and prominent negative anomalies for high-field-strength elements (HFSE; Ti, Hf, Zr, Nb and Ta). The depletion of the HFSE are characteristic of subduction-related magmas and may be attributed to crustal contamination and are thought to be resulted from the relative enrichment of mantle source by the influx of LILE from the dehydrating slab (e.g., McCulloch and Gamble 1991). Furthermore, the studied granitic rocks show prominent negative P anomalies (Fig. 13b) suggesting the influence of apatite and/or hornblende in the fractionating assemblages of granitic magmas.

Fig. 13: (a) Chondrite-normalized REE patterns for the studied granitic rocks. Normalizing values are from Sun and McDonough (1989). Fields of the calc-alkaline suite in the ANS are adapted from Azer (2013). (b) N-MORB normalized multielement patterns of trace elements in the studied granitic rocks. N-MORB concentrations from Sun and McDonough (1989).



\section{DISCUSSION}

\section{Magma types and constraints on potential sources}

Based on the aluminum saturation index and the mineral composition of the analyzed biotite, the studied granitic rocks exhibi an increase in alkaline character from tonalites to granodiorites. The relatively low agpaitic index $(<1)$ for the studied AGR evidences their calc-alkaline nature and they are 
Geochemistry and petrogenesis of the calc-alkaline Granitoids

related to I-type granite (Figs.14a and b). Furthermore, the studied AGR are magnesian and have low $10^{4 *} \mathrm{Ga} / \mathrm{Al}$ ranging from 1.82 to 2.28 with an average 2.05 , which is comparable with the average of I-type granite.

The nature of the parent melts forming the granitic magmas can be obtained from the leastdifferentiated samples of the granitic suites (e.g., Kessel et al. 1998; Mushkin et al. 2003). For the studied AGR, tonalites represent the least-evolved granitic rocks (e.g., the less siliceous granitic samples). They have high $\mathrm{Ba}$ and $\mathrm{Sr}$ concentrations, and their REE patterns are characterized by concave-upward patterns without significant Eu anomalies (Fig. 13a). These features seem to indicate a plagioclase (and garnet)poor, but amphibole rich sources (e.g., Bacon and Druitt 1988; Grove and Donnelly-Nolan 1986; Hildreth and Moorbath 1988; Koprubasi and Aldanmaz 2004). Wyllie (1984), introduces experimental evidence, for the generation of granitic magmas from the dehydration melting of metabasaltic sources by AFC processes toward more felsic compositions. Moreover, Douce and McCarthy (1998), introduced similar experiments suggesting that the dehydration melting of K-rich metabasaltic sources yield strongly peraluminous melts, which is in contrast with the predominantly metaluminous nature of the studied AGR. Furthermore, they are medium-K type (Fig. 10a), therefore, partial melting of the solely metabasaltic rock is not a suitable source for the studied AGR, because metabasaltic rocks have low $\mathrm{K}_{2} \mathrm{O}$ contents (e.g., Rapp and Watson 1995; Roberts and Clemens 1993).

The medium-K contents and high Fe/Mg ratios of the studied AGR (Fig. 10a, Table 6), indicated that their primary liquid source should be most likely a metaluminous protolith. Moreover, they are characterized by high abundances incompatible trace elements (LILE, HFSE) with slightly negative $\mathrm{Nb}$ and $\mathrm{Ta}$ anomalies, which could not be explained by direct derivation from crustal protoliths. The combined negative $\mathrm{Nb}$-Ta and $\mathrm{Zr}$-Hf anomalies (Fig. 13b) and the relatively high values of $\mathrm{Th} / \mathrm{Ta}, \mathrm{La} / \mathrm{Nb}$, and $\mathrm{Ba} / \mathrm{Th}$ (Table 6) indicate derivation of these granitoids from subduction-modified mantle source (e.g., Beyth et al. 1994; Jarrar et al. 2008; Kessel et al. 1998; Mushkin et al. 2003; Stern and Voegeli 1987).



Fig. 14: Discrimination diagrams for the studied granitic rocks (a) $\mathrm{K}_{2} \mathrm{O}$ versus $\mathrm{Na}_{2} \mathrm{O}$ (Collins et al. 1982) (b) $10^{4} \times \mathrm{Ga} / \mathrm{Al}$ versus $\mathrm{FeO} * / \mathrm{MgO}$ (Whalen et al. 1987). The insect diagram shows the variation of $\mathrm{SiO}_{2}$ versus $\mathrm{FeO}^{*} /\left(\mathrm{FeO}^{*}+\mathrm{MgO}\right)$ (Frost et al. 2001).

\section{Tectonic setting}

I-type (subduction-related) and S-type granites (collision-related) were identified by Chappel and White (1974). Later, the A-type (Collins et al. 1982) and M-type granites (Whalen et al. 1987) were recognized to accommodate within-plate granites and granites generated in oceanic arc tectonic environments, respectively. Pearce et al. (1984) suggested several diagrams for discriminating a variety of tectonic settings of granites. The studied AGR plot in the fields of syn-collision (Syn-COLG) and volcanic arc granites (VAG) (Figs. $15 \mathrm{a}$ and $\mathrm{b}$, respectively). In addition, based on the tectonic settings of the granitic rocks proposed by Maniar and Piccoli (1989; Fig. 7), the studied AGR samples plotted in the overlapped fields of calc-alkaline granite (CAG) and island arc granite (IAG).

The plots of the studied granitic rocks on the AFM diagram could be used to differentiate between compressional and tensional suites (Fig. 16). The investigated AGR samples are arranged on trend that tends to be nearly perpendicular to the F-M sideline for the entire trend pointing to compressional environment suggested by Petro et al. (1979). 
Fig. 15: (a) $\mathrm{Nb}$ versus $\mathrm{Y}$ (Pearce et al. 1984). (b) $\mathrm{Rb}$ versus $\mathrm{Y}+\mathrm{Nb}$ (Pearce, 1996). The shaded area points to the compositions of post-collision granitoids from Küster and Harms (1998).

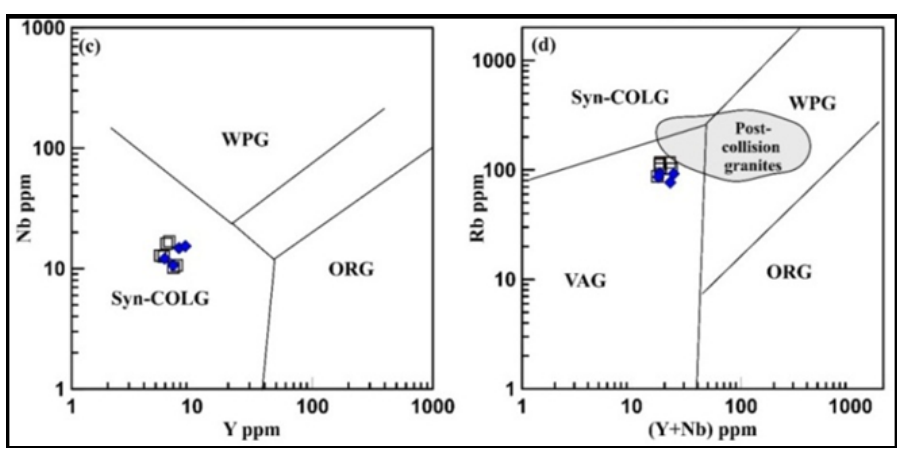

The chemistry of the analyzed plagioclase indicates that the AGR have relatively high values of $\mathrm{CaO} /\left(\mathrm{Na}_{2} \mathrm{O}+\mathrm{K}_{2} \mathrm{O}\right)$ ranging from 0.25 to 1.09 (Table 3), which indicate calc-alkaline nature (Macdonald et al. 1987). In addition, Nachit et al. (1985) used the composition of biotite as an indicator for the nature of original parent magmas. They concluded that the chemical composition of biotite could discriminate among peraluminous $(\mathrm{P})$, calc-alkaline $(\mathrm{C})$, subalkaline $(\mathrm{SA})$ and alkaline-peralkaline (A-PA) granites. The analyzed biotites plot in the calc-alkaline and subalkaline fields. Moreover, Abdel-Rahman (1994), suggested that the nature of the parental magmas from which the granitic rocks were originated could be assigned according to the composition of their igneous biotite. Moreover, Abdel-Rahman (1994) distinguished that, the biotite in alkaline (mostly anorogenic extension-related) suites including A-type granites are mostly iron-rich siliceous annite (av. $\mathrm{FeO} / \mathrm{MgO}$ ratio of 7.04 ), whereas those in peraluminous suites are siderophyllitic in composition (av. $\mathrm{FeO}^{*} / \mathrm{MgO}$ ratio of 3.48), whereas those in calc-alkaline, subduction-related orogenic suites are moderately enriched in $\mathrm{MgO}$, with an average $\mathrm{FeO} * \mathrm{MgO}$ ratio of 1.76 . The $\mathrm{FeO} * \mathrm{MgO}$ ratio of the analyzed biotite from AGR ranges from 1.5 to 4.59 with an average of 2.96, which is similar to the composition of biotite from calc-alkaline, subductionrelated orogenic suite (Fig. 4d). Moreover, the $\mathrm{Fe} /(\mathrm{Fe}+\mathrm{Mg}$ ) ratios of biotites from the studied AGR (0.460.62; Table 2). Anderson et al (1980) suggested that the $\mathrm{Fe} /(\mathrm{Fe}+\mathrm{Mg})$ ratios of biotite depend on the oxygen fugacity. In a high $\mathrm{fO}_{2}$, biotite can be oxidized and becomes rich in $\mathrm{Mg}$. Therefore, the Mg-rich biotite of the investigated AGR reflects low oxygen fugacity during their formation. In addition, the high $\mathrm{MgO}$ contents of biotite from the studied AGR suggest a high temperature of crystallization (Klob 1970).



The investigated I-type AGR are mainly granodiorite with subordinate tonalite, displaying metaluminous, calc-alkaline geochemical characteristics, and characterized by the scarcity of alkali feldspar, higher An contents, and higher mafic and iron-oxides contents. Wadi El-Akhder granitic rocks are depleted in $\mathrm{SiO}_{2}, \mathrm{~K}_{2} \mathrm{O}, \mathrm{Rb}, \mathrm{Pb}, \mathrm{Cr}$ and have high proportions of $\mathrm{MgO}, \mathrm{FeO}^{*}, \mathrm{TiO}_{2}, \mathrm{CaO}, \mathrm{P}_{2} \mathrm{O}_{5}$ and $\mathrm{Sr}$ contents. This suggests a mantle origin for the AGR as supported by the low ${ }^{87} \mathrm{Sr} /{ }^{86} \mathrm{Sr}$ initial ratios $(0.7029 \pm 0.0008)$ in Sinai (Brelski 1982). Based on these characteristics, it is believed that these rocks are subduction-related granites resultant from partial melting of mafic source, leaving garnet- or amphibolerich residues (Arth and Hanson 1975). A correlation was found between the depth of origin and the $\mathrm{Sr} / \mathrm{Rb}$ ratio (Condie 1973). This feature is explained as a consequence of Sr fractionation between solid and liquid phases. Higher $\mathrm{Sr} / \mathrm{Rb}$ ratios are an outstanding feature for greater depth (Condie 1973). The studied AGR have high $\mathrm{Sr} / \mathrm{Rb}$ ratio (av. 8.76) indicating that magma formation started at intermediary or even 
Geochemistry and petrogenesis of the calc-alkaline Granitoids

greater depths (>40 km; El-Tokhi et al. 2009). Furthermore, the multi-element spider plots could be used as an effective indicator for the discriminating the tectonic setting of the Egyptian granitoids rocks, especially the calc-alkaline (I-type) ones (Moghazi 2002). It is believed that the Egyptian calc-alkaline trondhjemite-tonalite-granodiorite suites were formed during the pre-collision $(\operatorname{arc})$ stage between 715 and $685 \mathrm{Ma}$ and prevailed during the collision stage between 665 and $614 \mathrm{Ma}$ (Greiling et al. 1994; Stern and Hedge 1985). The multi-element spider plots normalized to N-MORB for the studied AGR (Fig. 13b), are generally more akin to those of the pre-collision (arc) stage (I-type) granites. However, they exhibit slightly higher $\mathrm{Sr}, \mathrm{K}, \mathrm{Rb}$ and $\mathrm{Ba}$ contents, approaching those of the collision stage granites from the Eastern Desert of Egypt and possibly reflecting a more advanced stage of the arc formation. Accordingly, the studied granites are typical examples for I-type granites of the ANS (Fig 10a).

\section{Petrogenetic considerations}

\section{Fractional crystallization}

The studied granitic rock exhibiting well-defined negative correlation trend of silica with some major oxides and trace elements contents (Fig. 6). This trend could be resulted from fractional crystallization of major and minor phases during the evolution of both granitic suites. The observed systematic variations of trace elements ( $\mathrm{Rb}, \mathrm{Ba}, \mathrm{Sr}$ and $\mathrm{Y})$ from tonalite to granodiorite in the studied AGR (Fig. 11) are ascribed by increasing fractionation of plagioclase and K-feldspar, accompanied by amphibole, biotite, and accessory phases, such as titanite, apatite, zircon, and allanite (Fig. 11; Table 5). Such a fractionation trend is also suggested by the variation in modal compositions that define magmatic differentiation from tonalite to granodiorite in the AGR (Figs. 5a and b). In addition, the REE chondrite-normalized pattern (Figs. 13a and $b$ ) for each of the studied granitic types are similar and exhibiting LREE enrichment with HREE depletion patterns indicating the influence of the fractional crystallization in the studied granitic types. Moreover, the absence of pronounced $\mathrm{Eu}$ anomalies in the AGR samples coupled with the concaveupward REE profiles (Fig.8a), which resulting from depletion of the middle REE (Gd to Er) relative to the other HREE could be attributed to the fractionation of hornblende, which is the major constituent for the MREE. In addition, the analyzed samples from AGR exhibiting a negative correlation between $\mathrm{Y}$ and $\mathrm{SiO}_{2}$ (Fig. 6h), and show marked negative anomalies of $\mathrm{Y}$ in the multi-element spider diagrams normalized to N-MORB (Fig. 8b), indicating fractionation of hornblende (Pearce and Norry 1979). On the other hand, the absence or small negative anomalies of $\mathrm{Eu}, \mathrm{Ba}$ and $\mathrm{Sr}$ (Fig. 13) coupled with a weak negative correlation of $\mathrm{Ba}$ and $\mathrm{Sr}$ with increasing $\mathrm{SiO}_{2}$ (Fig. 11) in the studied AGR suggest that the fractionation of plagioclase has no important role in the evolution of the AGR.

The studied granitic rocks show a negative correlation of $\mathrm{TiO}_{2}$ and $\mathrm{P}_{2} \mathrm{O}_{5}$ with increasing $\mathrm{SiO}_{2}(\mathrm{Fig}$ 6a; Table 6), which could be explained by the fractionation of titanomagnetite and apatite, respectively. Moreover, the depletion of $\mathrm{Zr}$ in the studied granitic rocks (Fig. 13b) may be due to the fractionation of accessory phases such as zircon and allanite. In addition, the studied granodiorites have higher $\mathrm{SiO}_{2}$ contents than those of tonalites, but the former have the lowest values of Ba (Fig. 11f) which may account for biotite fractionation at the final stage of the magmatic process for AGR.

\section{Evidence for magma mixing}

The petrographic investigations of the studied AGR revealed that not only the fractional crystallization played a role in the modification of their parental melts but also magma mixing is contributed. Wadi ElAkhder granitic rocks include both mafic and felsic enclaves of varying sizes, shapes and compositions as well as common textural evidence that is account for interaction of mafic and silicic magmas such as quartz ocelli and complex zoning in plagioclase (e.g., Ahmad 2011; Baxter and Feely 2002; Choe and Jwa 2004; Palivcova et al. 1995).

The geochemical data of the studied AGR shows that these rocks lack of pronounced Eu anomalies (Fig.13a) and their contents of $\mathrm{Sr}, \mathrm{Ba}$, and $\mathrm{Rb}$ have weak correlation with $\mathrm{SiO}_{2}$ (Fig. 11). These features indicate that fractional crystallization was not the most important compositional modification process and that chemical variations may have been largely blurred by accumulation and mixing of melts (Koprubasi and Aldanmaz 2004). The most important process in generating much of the geochemical variation of the studied AGR appears to be resulted from the variable degree of partial melting and degree of relative 


\section{Mogahed, M. M.}

mixing between mafic and felsic melts. In addition, tonalites and granodiorites of the studied AGR exhibiting a continuous trend of major-element variations, also support mixing of magmas formed by partial melting of mafic sources (e.g., Baxter and Feely 2002; Frost and Mahood 1987; Guffanti et al. 1996; Hibbard 1991; Reid et al. 1983).

The exchange of elements between the mafic melts and crustal contaminants during melting represents an important factor that may affect the element distribution of granitic melts (Miller and Mittlefehldt 1982; Mittlefehldt and Miller 1983). According to Sylvester (1989) and Jung and Pfänder (2007), the granitic melt generated from crustal sources of sedimentary origin tend to have lower $\mathrm{CaO} / \mathrm{Na}_{2} \mathrm{O}$ ratio $(<0.2)$ than melts produced by melting of mafic compositions because the anorthite component of plagioclase in most crustal lithologies is quite low. However, derivation of granitic compositions from either crustal melts or more mafic mantle melts and subsequent fractionation of likely mineral assemblages will create similar trends that are characterized by an increase of the $\mathrm{CaO} / \mathrm{Na}_{2} \mathrm{O}$ ratio with an increasing silica content of the melt. The same conclusion will be reached when considering the variation in the $\mathrm{Al}_{2} \mathrm{O}_{3} / \mathrm{TiO}_{2}$ ratio. The studied AGR show high $\mathrm{CaO} / \mathrm{Na}_{2} \mathrm{O}$ ratios $(0.82-1.54)$ being a decrease from tonalite to granodiorites, they have also high $\mathrm{Al}_{2} \mathrm{O}_{3} / \mathrm{TiO}_{2}$ ratios (16.69-27.61) increasing toward the higher silica granitic type (granodiorites). These features indicate that emplacement of these granitoids was strongly affected by magma mixing of felsic and mafic melts.

High-alumina granitoids can result either from the melting of a psammite (graywacke) source or from the mixing of basaltic melt with granitic melt produced from metapelites (Sylvester 1998). Considering the studied AGR, the mixing of mafic and felsic sources are supported by the high total contents of $\mathrm{FeO}^{*}$, $\mathrm{MgO}$, and $\mathrm{TiO}_{2}$ in the tonalite (6.81-7.224 wt.\%), granodiorite (4.55-7.18 wt.\%), which are relatively higher (Table 6) than those in granitoids melted out from a pure metapelitic source (Fig. 12e). Such effects can best be observed in the variations of major oxide ratios such as $\mathrm{CaO} / \mathrm{Na}_{2} \mathrm{O}$ and $\mathrm{Al}_{2} \mathrm{O}_{3} / \mathrm{TiO}_{2}$ between mantle-derived mafic melts and crustal protoliths (Fig. 12a). The compositional points of AGR members (tonalites and granodiorites) are plotted close to the line of mixing of melts of different compositions in the $\mathrm{CaO} / \mathrm{Na}_{2} \mathrm{O}-\mathrm{Al}_{2} \mathrm{O}_{3} / \mathrm{TiO}_{2}$ and $\left(\mathrm{FeO} *+\mathrm{MgO}+\mathrm{TiO}_{2}\right)$ against $\mathrm{SiO}_{2}$ diagrams (Fig. 12a and e). In addition, all the studied granitic rocks are characterized by low $\mathrm{Rb} / \mathrm{Ba}(0.05-0.15)$ and $\mathrm{Rb} / \mathrm{Sr}(0.09-0.18)$ ratios and the location of their composition points in the field of sources depleted in clay minerals (Fig. 12f). Moreover, the plots of $\mathrm{Rb} / \mathrm{Sr} v \mathrm{vs}$. $\mathrm{Rb} / \mathrm{Ba}$ for the studied AGR exhibit a linear array of increasing $\mathrm{Rb} / \mathrm{Sr}$ with $\mathrm{Rb} / \mathrm{Ba}$ (Fig. 12b), supporting the effects of variation in crustal contamination of mantle-derived basaltic magma.

DePaolo (1981) stated that the ratio of $\mathrm{Th} / \mathrm{La}$ in granitic rocks could be used as indicator for the effects of crustal contamination of the magma derived from a component that is more mafic than the contaminant. The studied granitic rocks exhibit increasing ratio of $\mathrm{Th} / \mathrm{La}$ with increase in silica content (Fig. 17b), indicating the effects of crustal contamination of a magma derived from a component that is more mafic than the contaminant. The rocks from the AGR plot mostly along AFC trajectories drawn for $r=0.3$ and 0.5 . The results obtained from the variation of $\mathrm{Th} / \mathrm{La}$ ratios of the studied granitic rocks confirming the interpretation from both the major- and trace-element data (Fig. 12), which indicate the more pronounced effects of crustal contamination (or mixing) during the emplacement of the studied granitic rocks.
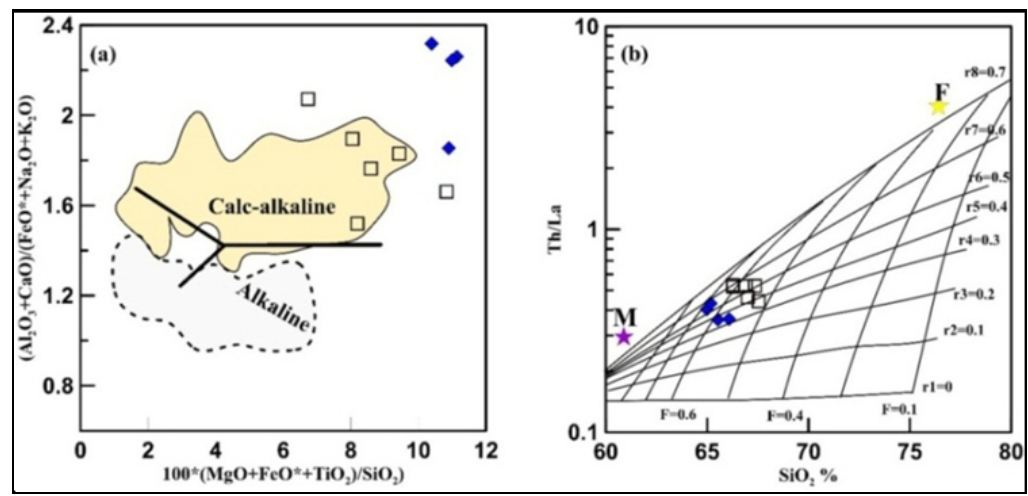

Fig. 17: (a) Discrimination diagram of Sylvester (1989); the calc-alkaline and alkaline fields of the ANS are adapted from Be'eri-Shlevin et al. (2009). (b) Modeling of the assimilation and fractional crystallization (AFC) process based on DePaolo (1981) and using the assimilationsensitive ratio of $\mathrm{Th} / \mathrm{La}$. Selected source and contaminant end-member compositions represent the average enriched mantlederived basaltic melts and average crustal composition, respectively (Aldanmaz et al. 2000 ). The $F$ value denotes the ratio of magma mass to original magma mass. 
Geochemistry and petrogenesis of the calc-alkaline Granitoids

\section{CONCLUSIONS}

The area of Wadi El-Akhder comprises syn-tectonic granitoids (AGR). Geochemically, the AGR are medium-K, calc-alkaline, metaluminous tonalite-granodiorite suite related to I-type granites that is formed during the pre-collision (arc) stage of the evolution of the ANS continental crust. The studied AGR are characterized by high abundances incompatible trace elements (LILE, HFSE) with slightly negative $\mathrm{Nb}$ and $\mathrm{Ta}$ anomalies, this could not be explained by direct derivation from crustal protoliths. The combined negative $\mathrm{Nb}-\mathrm{Ta}$ and $\mathrm{Zr}-\mathrm{Hf}$ anomalies and the relatively high values of $\mathrm{Th} / \mathrm{Ta}, \mathrm{La} / \mathrm{Nb}$, and $\mathrm{Ba} / \mathrm{Th}$ of the AGR refers to derivation from subduction-modified mantle source. AGR show high $\mathrm{CaO} / \mathrm{Na}_{2} \mathrm{O}$ ratios (0.82-1.54) being a decrease from tonalite to granodiorites, whereas they have also high $\mathrm{Al}_{2} \mathrm{O}_{3} / \mathrm{TiO}_{2}$ ratios (16.69-27.61) increasing toward the higher silica granitic type (granodiorites). These features indicate that emplacement of these granitoids were strongly affected by magma mixing of felsic and mafic melts.

\section{ACKNOWLEDGMENT}

Deep thanks to Dr. Galina M. Vovna, Far Eastern Geologic Institute, Russia, for the laboratory facilities during my post-doctoral scholarship. Many thanks for Dr. Karabtsov A. Alexandrovich and Ekimova N. Ivanovna (Far Eastern Geologic Institute, Russia), for their help and technical support with the electron microprobe analyses. Many thanks for Dr. Abd El Aziem Mehanna for his valuable recommendation and suggestion. Thanks for Dr. Mohammed M. Afife for his help in the field trips

\section{REFERENCES}

Abdel-Rahman, A.-F. M. (1994): Nature of biotites from alkaline, calc-alkaline, and peraluminous magmas. J. Petrol., 35, 525-541

Abdel-Rahman, A.-F. M. and El-Kibbi M. M. (2001): Anorogenic magmatism: chemical evolution of the Mount El-Sibai A-type complex (Egypt), and implications for the origin of within-plate felsic magmas. Geol. Mag., 138, 67-85

Ahmad, M. (2011): Enclaves in granitoids of north of Jonnagiri schist belt, Kurnool district, Andhra Pradesh: Evidence of magma mixing and mingling. J. Geol. Soc. India. 77, 557-573

Ahmed, A., El Sheshtawi Y. and El Tokhi M. (1993): Origin and geochemistry of Egyptian granitoid rocks in Nuweiba area, eastern Sinai. Journal of African Earth Sciences (and the Middle East). 17, 399413

Aldanmaz, E., Pearce J., Thirlwall M. and Mitchell J. (2000): Petrogenetic evolution of late Cenozoic, post-collision volcanism in western Anatolia, Turkey. J. Volcanol. Geotherm. Res., 102, 67-95

Anderson, J. L., Cullers R. L. and Van Schmus W. (1980): Anorogenic metaluminous and peraluminous granite plutonism in the Mid-Proterozoic of Wisconsin, USA. Contrib. Mineral. Petrol., 74, 311-328

Arth, J. G. and Hanson G. N. (1975): Geochemistry and origin of the early Precambrian crust of northeastern Minnesota. Geochim. Cosmochim. Acta. 39, 325-362

Avigad, D. and Gvirtzman Z. (2009): Late Neoproterozoic rise and fall of the northern Arabian-Nubian Shield: the role of lithospheric mantle delamination and subsequent thermal subsidence. Tectonophysics. 477, 217-228

Avigad, D., Sandler A., Kolodner K., Stern R., McWilliams M., Miller N. and Beyth M. (2005): Massproduction of Cambro-Ordovician quartz-rich sandstone as a consequence of chemical weathering of Pan-African terranes: Environmental implications. Earth Planet. Sci. Lett., 240, 818-826

Azer, M. K. (2013): Late Ediacaran (605-580 Ma) post-collisional alkaline magmatism in the ArabianNubian Shield: a case study of Serbal ring-shaped intrusion, southern Sinai, Egypt. J. Asian Earth Sci., 77, 203-223

Bacon, C. R., Druitt T. H. (1988): Compositional evolution of the zoned calcalkaline magma chamber of Mount Mazama, Crater Lake, Oregon. Contrib. Mineral. Petrol., 98, 224-256

Barker, F., Arth J.G. (1976): Generation of trondhjemitic-tonalitic liquids and Archean bimodal trondhjemite-basalt suites. Geology. 4, 596-600

Baxter, S. and Feely M. (2002): Magma mixing and mingling textures in granitoids: examples from the Galway Granite, Connemara, Ireland. Mineral. Petrol., 76, 63-74 


\section{Mogahed, M. M.}

Be'eri-Shlevin, Y., Katzir Y., Whitehouse M. (2009): Post-collisional tectonomagmatic evolution in the northern Arabian-Nubian Shield: time constraints from ion-probe U-Pb dating of zircon. J. Geol. Soc., $166,71-85$

Bentor, Y. (1985): The crustal evolution of the Arabo-Nubian Massif with special reference to the Sinai Peninsula. Precamb. Res., 28, 1-74

Beyth, M., Stern R. J., Altherr R. and Kröner A. (1994): The late Precambrian Timna igneous complex, southern Israel: evidence for comagmatic-type sanukitoid monzodiorite and alkali granite magma. Lithos. 31, 103-124

Bielski, M., Jäger E. and Steinitz G. (1979): The geochronology of Iqna granite (Wadi Kid pluton), southern Sinai. Contrib. Mineral. Petrol., 70, 159-165

Brelski, M. (1982): Stages in the evolution of the Arabian-Nubian Massif in Sinai. Hebrew Univ. Jerusalem

Brown, G. (2015): Calc-alkaline magma genesis: the Pan-African contribution to crustal growth. Evolution and Mineralization of the Arabian-Nubian Shield, King Abdulaziz Univ. Inst. Appl. Geol. Bull. 3, 19-29

Chappel, B., White A. (1974): Two contrasting granite types. Pac. Geol., 8, 173-174

Choe, W.-H., Jwa Y.-J. (2004): Petrological and geochemical evidences for magma mixing in the Palgongsan Pluton. Geosci. J., 8, 343

Collins, W., Beams S., White A. and Chappell B. (1982): Nature and origin of A-type granites with particular reference to southeastern Australia. Contrib. Mineral. Petrol., 80, 189-200

Condie, K. C. (1973): Archean magmatism and crustal thickening. Geol. Soc. Am. Bull., 84, 2981-2992

Condie, K. C. (1993): Chemical composition and evolution of the upper continental crust: contrasting results from surface samples and shales. Chem. Geol., 104, 1-37

Deer, W., Howie R., Zussman J. (1992) An introduction to the rock-forming minerals. 2nd ed. edn. Harlow, Essex, England : New York, NY : Longman Scientific \& Technical

DePaolo, D. J. (1981): Trace element and isotopic effects of combined wallrock assimilation and fractional crystallization. Earth Planet. Sci. Lett., 53, 189-202

Dixon, T. H. (1981): Gebel Dahanib, Egypt: a Late Precambrian layered sill of komatiitic composition. Contrib. Mineral. Petrol., 76, 42-52

Douce, A. E. P., McCarthy T. (1998) Melting of crustal rocks during continental collision and subduction. In: When continents collide: geodynamics and geochemistry of ultrahigh-pressure rocks. Springer, 27-55.

El-Mettwaly, A., Mashaal S. (1989): Geochemistry and tectonic environment of Old Granites of Wadi Ager, Southwest Sinai, Egypt. Mans. Sci. Bull., 91, 115-135

El-Mettwaly, A., Zalata A., El-Enen M.A. (1992): The evolution of the Pan-African granitoid rocks: geochemical evidences from SW Sinai massif, Egypt. Journal of African Earth Sciences (and the Middle East). 14, 111-119

El-Sheshtawi, Y., Dardir A., Khyamy A. (1993): Petrography and geochemistry of granitic rocks, their origin and tectonic environment in the Wadi Risasa area, Southeastern Sinai, Egypt. Journal of African Earth Sciences (and the Middle East). 17, 497-511

El-Tokhi, M., Musallum A., Amin B. (2009): Origin and geochemistry of Pan-African granitoid rocks in the Gabal Um Shomer area, Southwestern Sinai, Egypt. Chin. J. Geochem., 28, 125

Frost, B. R., Barnes C. G., Collins W. J., Arculus R. J., Ellis D. J., Frost C. D. (2001): A geochemical classification for granitic rocks. J. Petrol., 42, 2033-2048

Frost, T. P. and Mahood G. A. (1987): Field, chemical, and physical constraints on mafic-felsic magma interaction in the Lamarck Granodiorite, Sierra Nevada, California. Geol. Soc. Am. Bull., 99, 272-291

Furnes, H., El-Sayed M., Khalil S. and Hassanen M. (1996): Pan-African magmatism in the Wadi El-Imra district, Central Eastern Desert, Egypt: geochemistry and tectonic environment. J. Geol. Soc., 153, 705-718

Gass, I. (1981): Pan-African (upper Proterozoic) plate tectonics of the Arabian-Nubian shield. Dev. Precambrian Geol., 4, 387-405

Genna, A., Nehlig P., Le Goff E., Guerrot C. and Shanti M. (2002): Proterozoic tectonism of the Arabian Shield. Precamb. Res., 117, 21-40

Girardeau, J. and Mevel C. (1982): Amphibolitized sheared gabbros from ophiolites as indicators of the evolution of the oceanic crust: Bay of Islands, Newfoundland. Earth Planet. Sci. Lett., 61, 151-165 
Geochemistry and petrogenesis of the calc-alkaline Granitoids

Gokhale, N. (1968): Chemical composition of biotites as a guide to ascertain the origin of granites. Bull. Geol. Soc. Finland. 40, 107-111

Greiling, R. et al. (1994): A structural synthesis of the Proterozoic Arabian-Nubian Shield in Egypt. Geol. Rundsch., 83, 484-501

Grove, T. and Donnelly-Nolan J. (1986): The evolution of young silicic lavas at Medicine Lake Volcano, California: implications for the origin of compositional gaps in calc-alkaline series lavas. Contrib. Mineral. Petrol., 92, 281-302

Guffanti, M., Clynne M.A., Muffler L.P. (1996): Thermal and mass implications of magmatic evolution in the Lassen volcanic region, California, and minimum constraints on basalt influx to the lower crust. Journal of Geophysical Research: Solid Earth. 101, 3003-3013

Hassan, M. A. and Hashad A. H. (1990): Precambrian of Egypt. In: Said, R. (Ed.), The Geology of Egypt. . Balkema, Rotterdam.201-245

Hawthorne, F. C., Oberti R., Harlow G. E., Maresch W. V., Martin R. F., Schumacher J. C. and Welch M. D. (2012): Nomenclature of the amphibole supergroup. Am. Mineral., 97, 2031-2048

Heikal, M. A., Attawiya M.Y.A., El-Sheshtawi Y.A. (1985): Textural Patterns, geochemistry and origin of the granitoid rocks around Wadi El-Sheikh, Southwestern Sinai, Egypt. . Annals Geological Survey of Egypt, . 15, 197-210.

Hibbard, M. (1991): Textural anatomy of twelve magma-mixed granitoid systems. In: Didier J, Barbarin B (eds) Enclaves and granite petrology. . Elsevier, Amsterdam. (Dev Petrol 13), 431-444

Hildreth, W. and Moorbath, S. (1988): Crustal contributions to arc magmatism in the Andes of central Chile. Contrib. Mineral. Petrol., 98, 455-489

Hollister, L. S., Grissom G. C., Peters E. K., Stowell H. H., Gisson V. B. (1987): Confirmation of the empirical correlation of $\mathrm{Al}$ in hornblende with pressure of solidification of calc-alkaline plutons. Am. Mineral., 72, 231-239

Hussein, A. A. A., Ali M. M., El Ramly M. (1982): A proposed new classification of the granites of Egypt. J. Volcanol. Geotherm. Res., 14, 187-198

Irvine, T., Baragar W. (1971): A guide to the chemical classification of the common volcanic rocks. Can. J. Earth Sci., 8, 523-548

Jarrar, G. H., Manton W., Stern R., Zachmann D. (2008): Late Neoproterozoic A-type granites in the northernmost Arabian-Nubian Shield formed by fractionation of basaltic melts. Chemie der ErdeGeochemistry. 68, 295-312

Johnson, P. R., Woldehaimanot B. (2003): Development of the Arabian-Nubian Shield: perspectives on accretion and deformation in the northern East African Orogen and the assembly of Gondwana. Geol. Soc. Lon. Spec. Pub., 206, 289-325

Jung, S., Pfänder J.r.A. (2007): Source composition and melting temperatures of orogenic granitoids: constraints from $\mathrm{CaO} / \mathrm{Na} 2 \mathrm{O}, \mathrm{A} 12 \mathrm{O} 3 / \mathrm{TiO} 2$ and accessory mineral saturation thermometry. Eur. J. Mineral., 19, 859-870

Kessel, R., Stein M., Navon O. (1998): Petrogenesis of Late Neoproterozoic dikes in the Northern Arabian-Nubian Shield: implications for the origin of A-type granites. Precambrian Res., 92, 195-213

Klob, H. (1970): Über das Vorkommen eines porphyrischen Granites im Raume Sandl-Karlstift-Liebenau bei Freistadt im oberösterreichischen Mühlviertel (Granit von Typ „Karlstift”). Tscherm. Mineral. Petrogr. Mitt., 14, 311-323

Koprubasi, N. and Aldanmaz E. (2004): Geochemical constraints on the petrogenesis of Cenozoic I-type granitoids in Northwest Anatolia, Turkey: evidence for magma generation by lithospheric delamination in a post-collisional setting. Int. Geol. Rev., 46, 705-729

Kroner, A., Eyal M. and Eyal Y. (1990): Early Pan-African evolution of the basement around Elat, Israel, and the Sinai Peninsula revealed by single-zircon evaporation dating, and implications for crustal accretion rates. Geology. 18, 545-548

Küster, D., Harms U. (1998): Post-collisional potassic granitoids from the southern and northwestern parts of the Late Neoproterozoic East African Orogen: a review. Lithos. 45, 177-195

Le Maitre, R.W. (1989) A classification of igneous rocks and glossary of terms: Recommendations of the International Union of Geological Sciences Subcommission on the Systematics of Igneous Rocks, Blackwell Oxford, 193. 


\section{Mogahed, M. M.}

Leake, B. E. et al. (1997): Nomenclature of amphiboles; report of the Subcommittee on amphiboles of the International Mineralogical Association Commission on New Minerals and Mineral Names. Eur. J. Mineral., 9, 623-651

Leake, B. E. et al. (2003): Nomenclature of amphiboles: additions and revisions to the International Mineralogical Association's 1997 recommendations. The Canadian Mineralogist. 41, 1355-1362

Maniar, P. D., Piccoli P. M. (1989): Tectonic discrimination of granitoids. Geol. Soc. Am. Bull.,101, 635-643

Mashaal, S., El-Metwally, A., Ahmed, F., Essawy, M., Ragab, A. (1999): Geochemistry and petrogenesis of the granitoid rocks, southeastern Sinai, Egypt. Egypt. J. Geol., 43, 231-255

McCulloch, M. T., Gamble, J. (1991): Geochemical and geodynamical constraints on subduction zone magmatism. Earth Planet. Sci. Lett., 102, 358-374

Miller, C. F., Mittlefehldt D. W. (1982): Depletion of light rare-earth elements in felsic magmas. Geology. $10,129-133$

Mittlefehldt, D. W., Miller C. F. (1983): Geochemistry of the Sweetwater Wash Pluton, California: Implications for "anomalous" trace element behavior during differentiation of felsic magmas. Geochim. Cosmochim. Acta. 47, 109-124

Miyashiro, A. (1978): Nature of alkalic volcanic rock series. Contrib. Mineral. Petrol., 66, 91-104

Moghazi, A.-K.M. (1999): Magma source and evolution of Late Neoproterozoic granitoids in the Gabal El-Urf area, Eastern Desert, Egypt: geochemical and Sr-Nd isotopic constraints. Geol. Mag., 136, 285-300

Moghazi, A. (2002): Petrology and geochemistry of Pan-African granitoids, Kab Amiri area, Egyptimplications for tectonomagmatic stages in the Nubian Shield evolution. Mineral. Petrol., 75, 41-67

Mohamed, F., Hassanen, M., Matheis, G., Shalaby, M. (1994): Geochemistry of the Wadi Hawashia granite complex, northern Egyptian Shield. J. Afr. Earth Sci., 19, 61-74

Mushkin, A., Navon O., Halicz L., Hartmann G., Stein M. (2003): The petrogenesis of A-type magmas from the Amram Massif, southern Israel. J. Petrol., 44, 815-832

Nachit, H., Razafimahefa N., Stussi J.M., Carron J.P. (1985): Composition chemique des biotites et typologie magmatique des granitoids. Comtes Rendus Hebdomadaires de l'Academie des Sci.. 301, 813-818

Nockolds, S. R. (1947): The relation between chemical composition and paragenesis in the biotite micas of igneous rocks. Am. J. Sci., 245, 401-420

Norman, M., Griffin, W., Pearson, N., Garcia, M., O'reilly, S. (1998): Quantitative analysis of trace element abundances in glasses and minerals: a comparison of laser ablation inductively coupled plasma mass spectrometry, solution inductively coupled plasma mass spectrometry, proton microprobe and electron microprobe data. J. Anal. At. Spectrom., 13, 477-482

Norman, M., Pearson N., Sharma A., Griffin W. (1996): Quantitative analysis of trace elements in geological materials by laser ablation ICPMS: instrumental operating conditions and calibration values of NIST glasses. Geostand. Newslett., 20, 247-261

Obiora, S., Ukaegbu V. (2010): Preliminary investigation of the petrogenesis and geotectonic setting of the Precambrian basement complex rocks around northcentral Nigeria using trace and rare-earth elements geochemistry. J Mining Geol. 46, 127-137

Palivcova, M., Waldhausrova J., Ledvinkova V. (1995): Ocelli in mafic rocks of granitic complexes. Krystalinikum. 22, 149-186

Patiño Douce, A.E., Johnston A.D. (1991): Phase equilibria and melt productivity in the pelitic system: implications for the origin of peraluminous granitoids and aluminous granulites. Contrib. Mineral. Petrol., 107, 202-218

Pearce, J. (1996) Sources and settings of granitic rocks: Episodes, 19, 120-125.

Pearce, J. A., Harris N.B., Tindle A.G. (1984): Trace element discrimination diagrams for the tectonic interpretation of granitic rocks. J. Petrol., 25, 956-983

Pearce, J. A., Norry M.J. (1979): Petrogenetic implications of Ti, Zr, Y, and Nb variations in volcanic rocks. Contrib. Mineral. Petrol., 69, 33-47

Petro, W. L., Vogel T. A., Wilband J. T. (1979): Major-element chemistry of plutonic rock suites from compressional and extensional plate boundaries. Chem. Geol., 26, 217-235

Rajesh, H. (2007): The petrogenetic characterization of intermediate and silicic charnockites in high-grade terrains: a case study from southern India. Contrib. Mineral. Petrol., 154, 591-606 
Geochemistry and petrogenesis of the calc-alkaline Granitoids

Rapp, R. P. and Watson E. B. (1995): Dehydration melting of metabasalt at 8-32 kbar: implications for continental growth and crust-mantle recycling. J. Petrol., 36, 891-931

Reid, J.B., Evans O.C., Fates D.G. (1983): Magma mixing in granitic rocks of the central Sierra Nevada, California. Earth Planet. Sci. Lett., 66, 243-261

Rickwood, P. C. (1989): Boundary lines within petrologic diagrams which use oxides of major and minor elements. Lithos. 22, 247-263

Rieder, M. et al. (1999): Nomenclature of the micas. Mineral. Mag., 63, 267-279

Roberts, M. P., Clemens J.D. (1993): Origin of high-potassium, calc-alkaline, I-type granitoids. Geology. $21,825-828$

Rollinson, H. R. (1993) Using geochemical data: evaluation, presentation, interpretation. 1st Edition edn. Routledge, London

Roobol, M., Ramsay C., Jackson N. and Darbyshire D. (1983): Late Proterozoic lavas of the Central Arabian Shield - evolution of an ancient volcanic arc system. J. Geol. Soc., 140, 185-202

Schmidt, M. W. (1992): Amphibole composition in tonalite as a function of pressure: an experimental calibration of the Al-in-hornblende barometer. Contrib. Mineral. Petrol., 110, 304-310

Stern, R., Manton W. (1987): Age of Feiran basement rocks, Sinai: implications for late Precambrian crustal evolution in the northern Arabian-Nubian Shield. J. Geol. Soc., 144, 569-575

Stern, R. J. (1985): The Najd fault system, Saudi Arabia and Egypt: A late Precambrian rift-related transform system? Tectonics. 4, 497-511

Stern, R. J. (1994): Arc-assembly and continental collision in the Neoproterozoic African orogen: implications for the consolidation of Gondwanaland. Annual Review of Earth Planet. Sci., 22, 319-351

Stern, R. J. and Gottfried D. (1986): Petrogenesis of a late Precambrian (575-600 Ma) bimodal suite in northeast Africa. Contrib. Mineral. Petrol., 92, 492-501

Stern, R. J., Gottfried D. and Hedge C. E. (1984): Late Precambrian rifting and crustal evolution in the Northeastern Desert of Egypt. Geology. 12, 168-172

Stern, R. J. and Hedge C. E. (1985): Geochronologic and isotopic constraints on late Precambrian crustal evolution in the Eastern Desert of Egypt. Am. J. Sci., 285, 97-127

Stern, R. J., Voegeli D.A. (1987): Geochemistry, geochronology, and petrogenesis of a Late Precambrian ( $590 \mathrm{Ma})$ composite dike from the North Eastern Desert of Egypt. Geol. Rundsch., 76, 325-341

Streckeisen, A. (1976): To each plutonic rock its proper name. Earth-Sci. Rev.. 12, 1-33

Streckeisen, A., Le Maitre R. (1979): A chemical approximation to the modal QAPF classification of the igneous rocks. Neues Jahrb. Mineral. Abh., 136, 169-206

Struchio, N., Sultan M., Sylvester P., Batiza R. (1982): Age and origin of Meatiq Dome: implications for the stratigraphy and tectonic history of the Central Eastern Desert of Egypt. Precamb. Res., 16, A57-A58.

Sun, S.-S. and McDonough W. (1989): Chemical and isotopic systematics of oceanic basalts: implications for mantle composition and processes. Geol. Soc. Lon. Spec. Pub., 42, 313-345

Sweatman, T., Long J. (1969): Quantitative electron-probe microanalysis of rock-forming minerals. J. Petrol., 10, 332-379

Sylvester, P. J. (1989): Post-collisional alkaline granites. The Journal of Geology. 97, 261-280

Sylvester, P. J. (1998): Post-collisional strongly peraluminous granites. Lithos. 45, 29-44

Taylor, S. (1965): The application of trace element data to problems in petrology. Phys. Chem. Earth., 6, $133-213$

Taylor, S. R., McLennan S. M. (1985) The continental crust: its composition and evolution. Blackwell Scientific Publications, United States

Taylor, S. R. and McLennan S. M. (1995): The geochemical evolution of the continental crust. Rev. Geophys., 33, 241-265

Whalen, J. B., Currie K. L. and Chappell B. W. (1987): A-type granites: geochemical characteristics, discrimination and petrogenesis. Contrib. Mineral. Petrol., 95, 407-419

Wyllie, P. J. (1984): Constraints imposed by experimental petrology on possible and impossible magma sources and products. Philosophical Transactions of the Royal Society of London. Series A, Mathematical and Physical Sciences. 310, 439-456. 
Mogahed, M. M.

جيوكيميائية وأصل نشأة صخور الجرانيتات الكلسي - قلوية في شمال الارع العربي النويي. حالة دراسية من جرانيتات وادي الأخضر ، جنوب سيناء، مصر

\section{مصطفى محمد مجاهد}

كلية العلوم - جامعة بنها

\section{الخلاصة}

تعد صخور الجرانيتات ذات النشأة النارية والني تكونت في مراحل قبل التصادم من حركة البان أفريقية البانية للجبال خلال

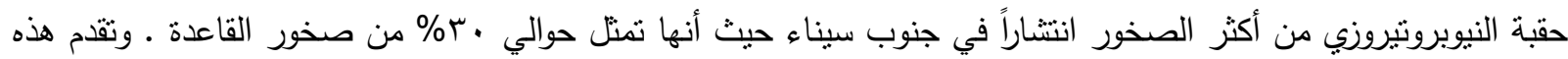
المخطوطة دراسات بترولوجية وجيوكيميائية بالإضافة الي تحليل كيميائي للمعادن الأساسية لصخور الجرانيتات القديمة

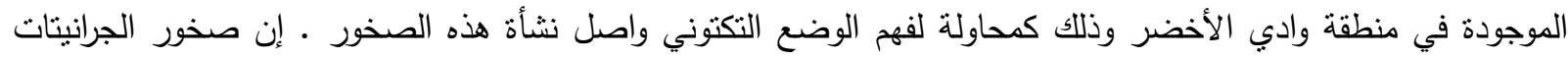
القديمة بمنطقة وادي الأخضر بجنوب غرب سيناء تتكون أساساً من صخور الجرانوديورايت بالإضافة الي صخور التوناليت. وهذه الصخور تحتوي على نسبة بوتاسيوم وألمونيوم متوسطة وتتتمي للصخور الكلسي-قلوية والتي تكونت في منطقة أقواس


والماغنسيوم والمنجنيز والكالسيوم وكذلك الاسترانشيوم والباريوم والفانديوم في حين انها تحنوي على نسب عالئي عالية من أكاسيد

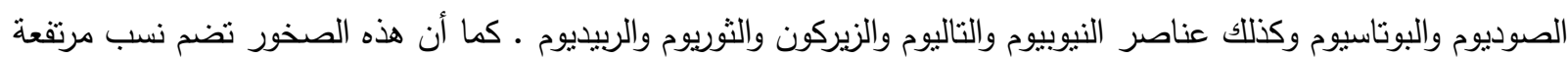

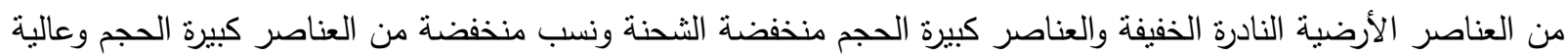

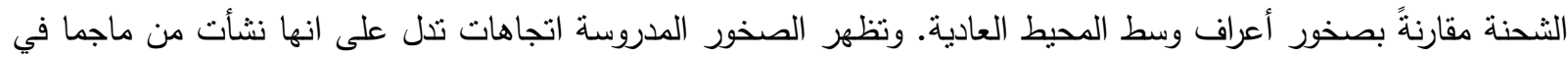
منطقة الوشاح تأثرت بعملية التمايز الصهيري وأنها تعرضت لمراحل من التلوث الصهيري أنثاء نطورها. وتؤكد هذه المخطوطة بأن الخصائص الكيميائية لصخور الجرانيتات المدروسة بمنطقة وادي الأخضر بجنوب سيناء تتتابه مع صخور جرانيتات الدرع العربي النوبي المتكونة بمناطق الانضواء. 